\title{
Composition-dependent transition in the magnetocrystalline anisotropy of tetragonal Heusler alloys $\operatorname{Rh}_{2} T \operatorname{Sb}(T=\mathrm{Fe}, \mathrm{Co})$
}

\author{
Gerhard H. Fecher $\odot,{ }^{*}$ Yangkun He $\odot$, and Claudia Felser \\ Max-Planck-Institute for Chemical Physics of Solids, D-01187 Dresden, Germany
}

(Received 8 September 2020; revised 11 March 2021; accepted 5 April 2021; published 10 May 2021)

\begin{abstract}
This paper reports on first-principles calculations of the electronic and magnetic structure of tetragonal Heusler compounds with the composition $\mathrm{Rh}_{2} \mathrm{Fe}_{x} \mathrm{Co}_{1-x} \mathrm{Sb}(0 \leqslant x \leqslant 1)$. It is found that the magnetic moments increase from 2 to $3.4 \mu_{B}$ and the Curie temperature decreases from 500 to $464 \mathrm{~K}$ with increasing Fe content $x$. The $3 d$ transition metals make the main contribution to the magnetic moments, whereas Rh contributes only approximately $0.2 \mu_{B}$ per atom, independent of the composition. The paper focuses on the magnetocrystalline anisotropy of the borderline compounds $\mathrm{Rh}_{2} \mathrm{FeSb}, \mathrm{Rh}_{2} \mathrm{Fe}_{0.5} \mathrm{Co}_{0.5} \mathrm{Sb}$, and $\mathrm{Rh}_{2} \mathrm{CoSb}$. A transition from easy-axis to easy-plane anisotropy is observed when the composition changes from $\mathrm{Rh}_{2} \mathrm{CoSb}$ to $\mathrm{Rh}_{2} \mathrm{FeSb}$. The transition occurs at an iron concentration of approximately $40 \%$. The difference in the anisotropy is caused by differences in the charge and spin distributions, mainly at $\mathrm{Fe}$ and $\mathrm{Co}$. The results of the calculations are in good agreement with recent experiments.
\end{abstract}

DOI: 10.1103/PhysRevMaterials.5.054404

\section{INTRODUCTION}

Permanent or hard magnets are made of bulk materials with strong anisotropy, which may be based on magnetocrystalline anisotropy, shape anisotropy, or both. In magnets with magnetocrystalline anisotropy, there should be only one easy crystal axis of magnetization so the anisotropy is uniaxial. Such an uniaxial magnetocrystalline anisotropy is found, for example, in tetragonal or hexagonal systems. Heusler alloys are compounds with formula $T_{2} T^{\prime} M$, where $T$ and $T^{\prime}$ are transition metals, and $M$ is a main group element. Some of these compounds and alloys crystallize in tetragonal structure; however, most of them have a cubic crystal structure. One advantage of Heusler compounds is that most of them do not contain rareearth elements; rather, the magnetic properties are provided by $3 d$ transition metals. Many tetragonal Heusler alloys are Mn based and several exhibit structural martensite-austenite phase transitions. In particular, in the inverse structures with space group $I \overline{4} m 2$, the magnetic moments of the Mn atoms exhibit antiparallel coupling. Thus, these alloys are generally ferrimagnets with low saturation magnetisation. The $\mathrm{Rh}_{2} T M$ alloys $\left(T^{\prime}=\mathrm{V}, \mathrm{Mn}, \mathrm{Fe}, \mathrm{Co} ; M=\mathrm{Sn}, \mathrm{Sb}\right)$ crystallize in a regular tetragonal structure with space group $I 4 / \mathrm{mmm}$ and are expected to exhibit uniaxial anisotropy when the $3 d$ transition metals have large moments.

\footnotetext{
*fecher@cpfs.mpg.de
}

Published by the American Physical Society under the terms of the Creative Commons Attribution 4.0 International license. Further distribution of this work must maintain attribution to the author(s) and the published article's title, journal citation, and DOI. Open access publication funded by the Max Planck Society.
Experiments on the crystal structure and magnetic properties of $\mathrm{Rh}_{2}$-based Heusler compounds were reported by Dhar et al. [1], who observed a tetragonal structure and a magnetic moment of $1.4 \mu_{B}$ in the primitive cell. A Curie temperature of approximately $450 \mathrm{~K}$ was measured. Further, Faleev et al. recently reported $a b$ initio calculations for many tetragonal Heusler compounds (including $\mathrm{Rh}_{2} \mathrm{FeSb}$ and $\mathrm{Rh}_{2} \mathrm{CoSb}$ ) [2]. This work proposed that thin films of $\mathrm{Rh}_{2} \mathrm{CoSb}$ exhibit uniaxial, perpendicular anisotropy with the easy direction along the $c([001])$ axis. Experiments and calculations both suggest that $\mathrm{Rh}_{2} \mathrm{CoSb}$ might be a suitable hard magnetic material with uniaxial anisotropy. However, the constituent elements, in particular Rh, might be too expensive for applications where bulk materials are needed, for example, permanent magnets in electric engines. However, the cost of the materials is not as important for thin film applications, for example, magnetic recording media or magnetoelectronic memory devices.

We recently reported experiments on the magnetic properties of $\mathrm{Rh}_{2} \mathrm{CoSb}$ and suggested its use as material for heat-assisted magnetic recording [3]. It was found that $\mathrm{Rh}_{2} \mathrm{CoSb}$ has uniaxial anisotropy, where $c$ is the easy axis. The present paper describes theoretically the magnetic properties of $\mathrm{Rh}_{2} \mathrm{CoSb}$, its sister compound $\mathrm{Rh}_{2} \mathrm{FeSb}$, and alloys with mixed $\mathrm{Co}_{1-x} \mathrm{Fe}_{x}$ composition. Details of the accompanying experiments are described in Ref. [3] and in the Supplemental Material [4].

\section{DETAILS OF THE CALCULATIONS}

The electronic and magnetic structures of $\mathrm{Rh}_{2} T \mathrm{Sb}(T=$ $\mathrm{Fe}, \mathrm{Co}$ ) were calculated using WIEN2k [5-7] and SPRKKR $[8,9]$ in the local spin-density approximation. In particular, the generalized gradient approximation of Perdew, Burke, and Ernzerhof [10] was used to parametrize the exchange correlation functional. A $k$ mesh based on $126 \times 126 \times 126$ points 


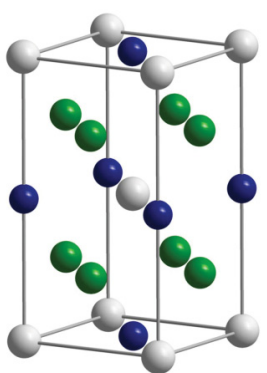

(a) ordered (regular)

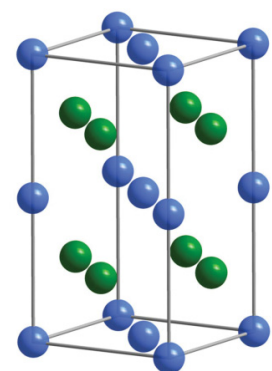

(b) disordered $(T \Leftrightarrow \mathrm{Sb})$

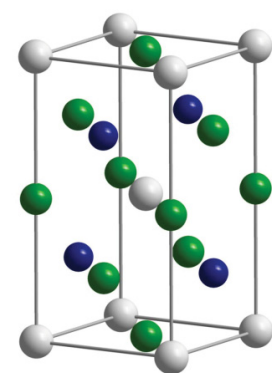

(c) ordered (inverse)
FIG. 1. Crystal structure of $\mathrm{Rh}_{2} \mathrm{TSb}(T=\mathrm{Fe}, \mathrm{Co})$. In the wellordered regular structure (a), the sites of the lattice with space group I 4/mmm (139) are occupied as follows: $4 d(01 / 21 / 4)$, Rh; $2 b(0$ $01 / 2), T$; and $2 a(000)$, Sb. In the disordered structure (b), the $T$ and $\mathrm{Sb}$ atoms are randomly distributed on the $2 \mathrm{~b}$ and $2 \mathrm{a}$ sites. The inverse tetragonal structure is shown in (c) for comparison.

of the full Brillouin zone was used for integration when the total energies were calculated to determine the magnetocrystalline anisotropy (see also Appendix D). The calculations are described in greater detail in Refs. [11,12]. The spin spirals and magnons were calculated according to the schemes described in Refs. [13,14], respectively. Calculations for the disordered or off-stoichiometric compounds with mixed site occupations were performed using SPRKKR and the coherent potential approximation (CPA) [15] in the full potential mode. The CPA allows the simulation of random site occupation by different elements. Complications arising in the calculation of the magnetic anisotropy energies are discussed in detail by Khan et al. [16], who compared results obtained using WIEN2k and SPRKKR.

The basic crystal structure of the tetragonal Heusler compounds [prototype $\mathrm{Rh}_{2} \mathrm{VSn}$; $t I 8 ; I 4 / \mathrm{mmm}$ (139) dba] [17] is shown in Fig. 1(a). The atoms are located in the ferromagnetic structure on the $4 d, 2 b$, and $2 a$ Wyckoff positions of the centered tetragonal cell. The magnetic order changes the symmetry, and the resulting magnetic space group for collinear ferromagnetic order with moments along the $c$ axis is $I 4 / \mathrm{mm}^{\prime} \mathrm{m}^{\prime}$ (139.537), where ' is the spin reversal operator [18]. The symmetry is reduced to that of space group $I m^{\prime} m^{\prime} m$ (71.536) when the magnetization $\vec{M}$ is along the $a$ axis ([100]) or $F m^{\prime} m^{\prime} m$ (69.524) for $\vec{M} \|[110]$.

The electronic structure and magnetic properties were calculated using the optimized lattice parameters. As a starting point, the lattice parameters of two alternative structures were optimized using WIEN2k. In addition to the regular Heusler structure described above, the inverse structure with space group $I \overline{4} m 2$ (119) $d b c a$ was assumed. In this structure, the positions of the Co atom and one of the Rh atoms are interchanged. Spin-orbit interaction was considered owing to the high $Z$ values of $R h$ and $S b$. Note that the spin-orbit interaction is an intrinsic property in the fully relativistic SPRKKR calculations, which solve the Dirac equation. The results of the optimization are summarized in Table I. The regular structure is found to have lower energy; it thus describes the ground state. The energy difference compared to the inverse structure is approximately $310 \mathrm{meV}$. The formation enthalpy is
TABLE I. Structural properties of $\mathrm{Rh}_{2} \mathrm{CoSb}$. Calculations are performed for the regular (139) and inverse (119) Heusler structures. The lattice parameters $(a, c, c / a)$, formation enthalpy $\left(\Delta H_{f}\right)$, and spin magnetic moment $m_{\mathrm{s}}$ of the primitive cell (total experimental magnetic moment) are listed. Experimental values from Ref. [1] are shown for comparison. Note that the magnetic moment in this reference is not saturated.

\begin{tabular}{lccccc}
\hline \hline & \multicolumn{2}{c}{ Calculated } & & \multicolumn{2}{c}{ Exp. } \\
\cline { 2 - 3 } \cline { 6 - 6 } & 139 & 119 & & Here [3] & {$[1]$} \\
\hline$a(\AA)$ & 4.0104 & 3.95 & & 4.0394 & 4.04 \\
$c(\AA)$ & 7.3628 & 7.56 & & 7.1053 & 7.08 \\
$c / a$ & 1.836 & 1.91 & & 1.759 & 1.75 \\
$\Delta H_{f}(\mathrm{meV})$ & -398 & -84 & & \\
$m_{\mathrm{s}}\left(\mu_{B}\right)$ & 2.04 & 1.79 & & 2.36 & 1.4 \\
$T_{C}(\mathrm{~K})$ & & & 450 & 450 \\
\hline \hline
\end{tabular}

calculated as

$$
\Delta H_{f}=\frac{1}{4}\left[E_{\mathrm{tot}}-\left(2 E_{\mathrm{Rh}}+E_{\mathrm{Co}}+E_{\mathrm{Sb}}\right)\right],
$$

that is, the difference between the total energy of the compound in different structures and the sum of the energies of the elements in their ground-state structure normalized by the number of atoms. The formation enthalpy is clearly lower for the regular tetragonal structure than for the inverse tetragonal structure (see Table I). Note that the formation enthalpy for the cubic $L 2_{1}$ structure is also negative $(\approx-60 \mathrm{meV})$, however, it is certainly above that of the tetragonal variants of the structure. The calculated lattice parameters are in good agreement with experimental values [1]; however, the calculated $c$ value and $c / a$ ratio are approximately $4 \%$ larger. This finding might be explained by either a temperature effect or some small disorder in the experiment.

\section{RESULTS AND DISCUSSION}

\section{A. Electronic and magnetic structure of $\mathrm{Rh}_{2} \mathrm{CoSb}$}

The calculated electronic structure of $\mathrm{Rh}_{2} \mathrm{CoSb}$ in the regular tetragonal Heusler structure is illustrated in Fig. 2 in terms of the band structure and density of states $[n(E)]$. The relativistic bands, spin-resolved total density of states, and its atomic contributions are shown. The electronic structure is calculated in the full relativistic mode by solving the Dirac equation. The band structure from semirelativistic calculations is shown in the Appendixes.

Both rhodium and cobalt contribute to the magnetic moment of the compound. The spin and orbital magnetic moments are $m_{s}^{\mathrm{Co}}=1.656 \mu_{B}$ and $m_{l}^{\mathrm{Co}}=0.139 \mu_{B}$ for cobalt and $m_{s}^{\mathrm{Rh}}=0.206 \mu_{B}$ and $m_{l}^{\mathrm{Rh}}=0.007 \mu_{B}$ for rhodium, respectively. The overall magnetic moment (spin plus orbital) of the primitive cell is $m_{\mathrm{tot}}=2.188 \mu_{B}$. The orbital moment of the Co atoms makes a remarkably large contribution.

The real-space charge and spin distributions are shown in Fig. 3. The charge density $[\sigma(r)]$ of the atoms has no striking shape. It appears to be nearly spherical but still reflects the two- or fourfold symmetry. As expected, most of the electrons are close to the ion cores. By contrast, the spin or 

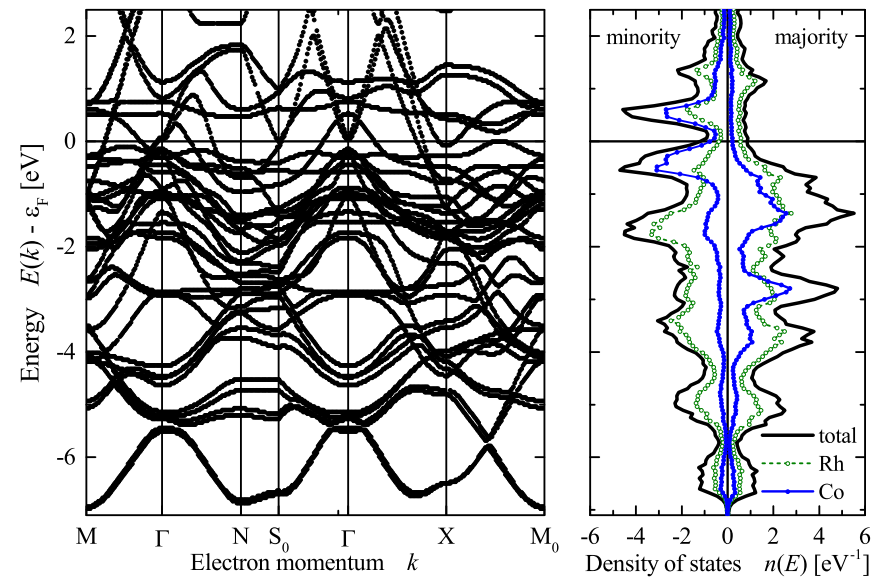

FIG. 2. Electronic structure of $\mathrm{Rh}_{2} \mathrm{CoSb}$ (I). Shown is the fully relativistic band structure together with the total and site ( $\mathrm{Rh}$ and $\mathrm{Co}$ ) specific, spin-resolved densities of states.

magnetization density $[\sigma(r)]$ has a much more pronounced shape, depending on the plane. In particular, in the (110) plane, it has a distinct butterfly shape. The spin density is positive at both the $\mathrm{Co}$ and $\mathrm{Rh}$ atoms. It is clearly higher near the $\mathrm{Co}$ atoms than near the $\mathrm{Rh}$ atoms, which ultimately gives Co a higher magnetic moment. The magnetization density of the $\mathrm{Rh}$ atoms is aligned along the magnetization direction and points somewhat toward the nearest Co atoms.

\section{Magnetic anisotropy}

Further, the directional dependence of the magnetization was investigated to explain the collinear magnetic order in detail. In particular, the total energy was calculated for cases where the magnetization points along different crystallographic directions. The obtained energy differences make it possible to determine the magnetocrystalline anisotropy (see also Appendix D).

In the magnetic anisotropy of $\mathrm{Rh}_{2} \mathrm{CoSb}$, the easy axis is along the $c([001])$ axis. The simple second-order uniaxial anisotropy constant is $K_{u}=1.37 \mathrm{MJ} / \mathrm{m}^{3}$ [see Eqs. (D1) and (D2) in Appendix D1]. This results in an anisotropy field of $\mu_{0} H_{u} \approx 2.4 \mathrm{~T}$. A more detailed analysis reveals that the simple second-order anisotropy constant $K_{u}$ is not sufficient to describe the magnetocrystalline anisotropy, as discussed in Sec. III D.

Further, the dipolar magnetocrystalline anisotropy was calculated as described in Appendix D3 and was found to be $\Delta E_{\text {dipaniso }}=0.09 \mu \mathrm{eV}$. The positive value indicates an easy dipolar direction along the [001] axis. The dipolar anisotropy is rather small compared to the anisotropy calculated from the total energy. Here, it was calculated for a sphere with a radius of $30 \mathrm{~nm}$. The results for other shapes will be different, resulting in a distinct shape anisotropy. In particular, in thin films, the dimension perpendicular to the film is much smaller than the dimensions in the film plane. Therefore, the summation in Eq. (D16) becomes a truncated sphere that is strongly anisotropic, and a pronounced thin film anisotropy appears. This thin film anisotropy will also be affected by the magnetic moments, which are different at interfaces and surfaces from that at the center layers of the film.
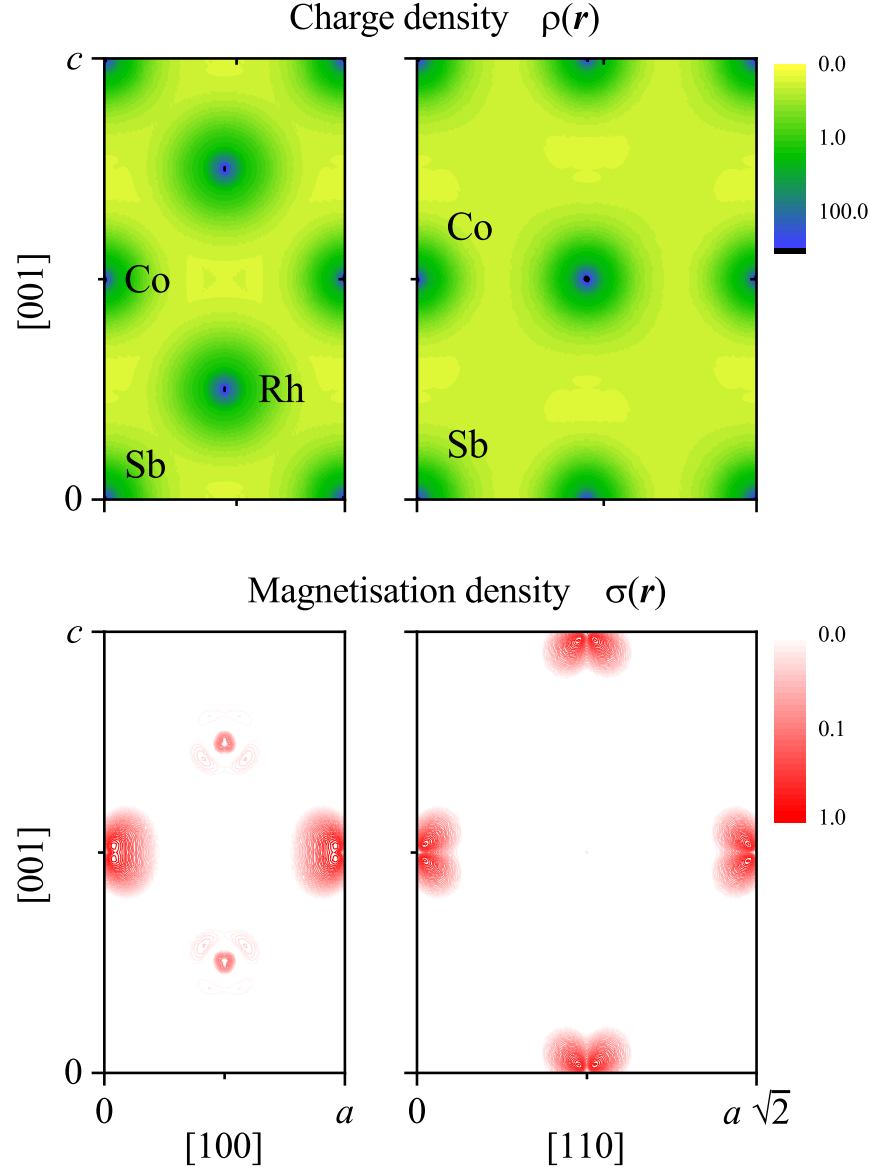

FIG. 3. Electronic structure of $\mathrm{Rh}_{2} \mathrm{CoSb}$ (II). Fully relativistic charge $[\rho(r)]$ and spin $[\sigma(r)]$ distributions for the (001) and (110) planes are shown. The calculation is for $m \| c$, that is, the magnetization points along [001] according to the easy axis behavior of the magnetic anisotropy. (Note: Color bars are in atomic units.)

\section{B. Results for $\mathrm{Rh}_{2} \mathrm{FeSb}$}

The calculations for $\mathrm{Rh}_{2} \mathrm{FeSb}$ were performed in the same way as for $\mathrm{Rh}_{2} \mathrm{CoSb}$. The regular structure with space group No. 139 was found to be more stable than the inverse structure with space group No. 119. Its calculated formation enthalpy amounts to $\Delta H_{f}=-226 \mathrm{meV}$. In addition, as in the case of $\mathrm{Rh}_{2} \mathrm{CoSb}$, the calculated $c$ lattice parameter, and thus $c / a$, are considerably larger than the experimental values (see Table II).

The electronic structure of $\mathrm{Rh}_{2} \mathrm{FeSb}$ is illustrated in Fig. 4. The fully relativistic band structure and the spin- and siteresolved densities of states are shown. The calculated spin and orbital magnetic moments are $m_{s}^{\mathrm{Fe}}=2.978 \mu_{B}$ and $m_{l}^{\mathrm{Fe}}=$ $0.080 \mu_{B}$ for iron and $m_{s}^{\mathrm{Rh}}=0.228 \mu_{B}$ and $m_{l}^{\mathrm{Rh}}=0.006 \mu_{B}$ for rhodium, respectively. The overall magnetic moment (spin plus orbital) of the primitive cell is $m_{\mathrm{tot}}=3.488 \mu_{B}$. The magnetic moment of the Fe atoms is strongly localized, which is typical of Heusler compounds with high magnetic moments. It clearly exceeds the value for elemental iron.

The real-space charge and spin distributions of $\mathrm{Rh}_{2} \mathrm{FeSb}$ are shown in Fig. 5. As in the Co-containing compound, $\sigma(r)$ does not have a pronounced shape (compare Fig. 3). The magnetization density $[\sigma(r)]$ around the Fe atoms has a less 
TABLE II. Structural properties of $\mathrm{Rh}_{2} \mathrm{FeSb}$. Calculations are performed for the regular tetragonal Heusler structures. Lattice parameters $(a, c, c / a)$ and spin magnetic moment $m_{\mathrm{s}}$ of the primitive cell are listed. Experimental values from Ref. [1] are shown for comparison. Note that the experimental moments in Ref. [1] are not saturated.

\begin{tabular}{lccc}
\hline \hline & & \multicolumn{2}{c}{ Experiment } \\
\cline { 3 - 4 } & Calculated & This paper & Ref. [1] \\
\hline$a(\AA)$ & 4.0418 & 4.0708 & 4.07 \\
$c(\AA)$ & 7.3995 & 6.9663 & 6.96 \\
$c / a$ & 1.8308 & 1.7112 & 1.71 \\
$m_{\mathrm{s}}\left(\mu_{B}\right)$ & 3.4 & 3.8 & 2.8 \\
$T_{C}(\mathrm{~K})$ & & 510 & 510 \\
\hline \hline
\end{tabular}

distinct shape compared to $\mathrm{Co}$ in $\mathrm{Rh}_{2} \mathrm{CoSb}$; it is also not greatly affected by changes in the magnetization direction. The main difference is the magnetization density around the $\mathrm{Rh}$ atoms, which is rotated and appears to be aligned along the magnetization direction.

Table III compares the calculated magnetic data of $\mathrm{Rh}_{2} \mathrm{CoSb}$ and $\mathrm{Rh}_{2} \mathrm{FeSb}$. $\mathrm{Rh}_{2} \mathrm{FeSb}$ clearly has a smaller orbital magnetic moment than $\mathrm{Rh}_{2} \mathrm{CoSb}$, whereas its spin magnetic moment is higher because of the effect of the Fe atoms. The induced magnetic moments of the Rh atoms are similar in both compounds.

The calculated Curie temperatures are of the same order of magnitude as the experimental values. In contrast to the calculated results, however, the experimental value of the $\mathrm{Fe}$ compound is higher than that of the Co compound. A possible reason is differences in the variation of the lattice parameters with temperature, which affect the exchange coupling parameters and thus $T_{C}$ and also the spin stiffness. Note that a much lower Curie temperature is obtained for the Co compound when it is off-stoichiometric (see Appendix B 2), whereas the $T_{C}$ value of the off-stoichiometric Fe compound is slightly higher.
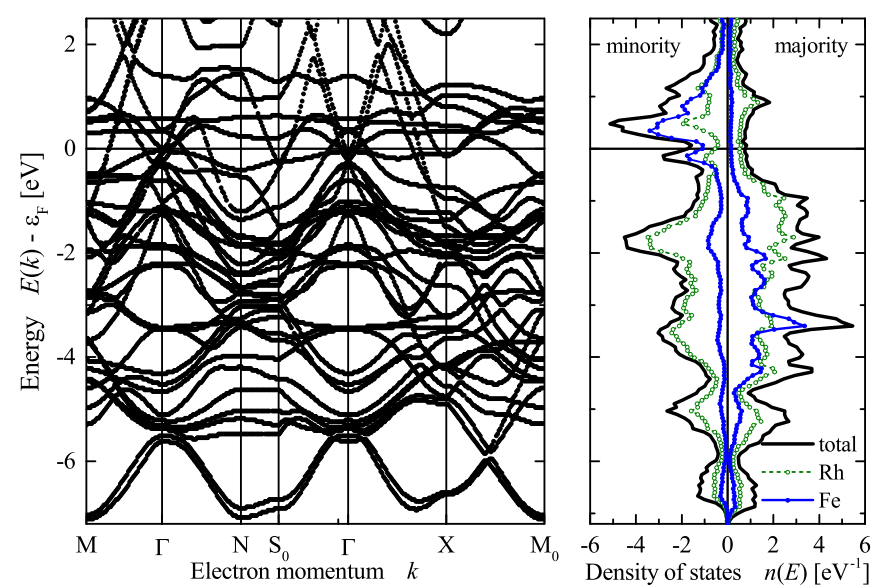

FIG. 4. Electronic structure of $\mathrm{Rh}_{2} \mathrm{FeSb}$ (I). Fully relativistic band structure is shown, along with the total- and site-specific spinresolved densities of states for $\mathrm{Rh}$ and $\mathrm{Fe}$.
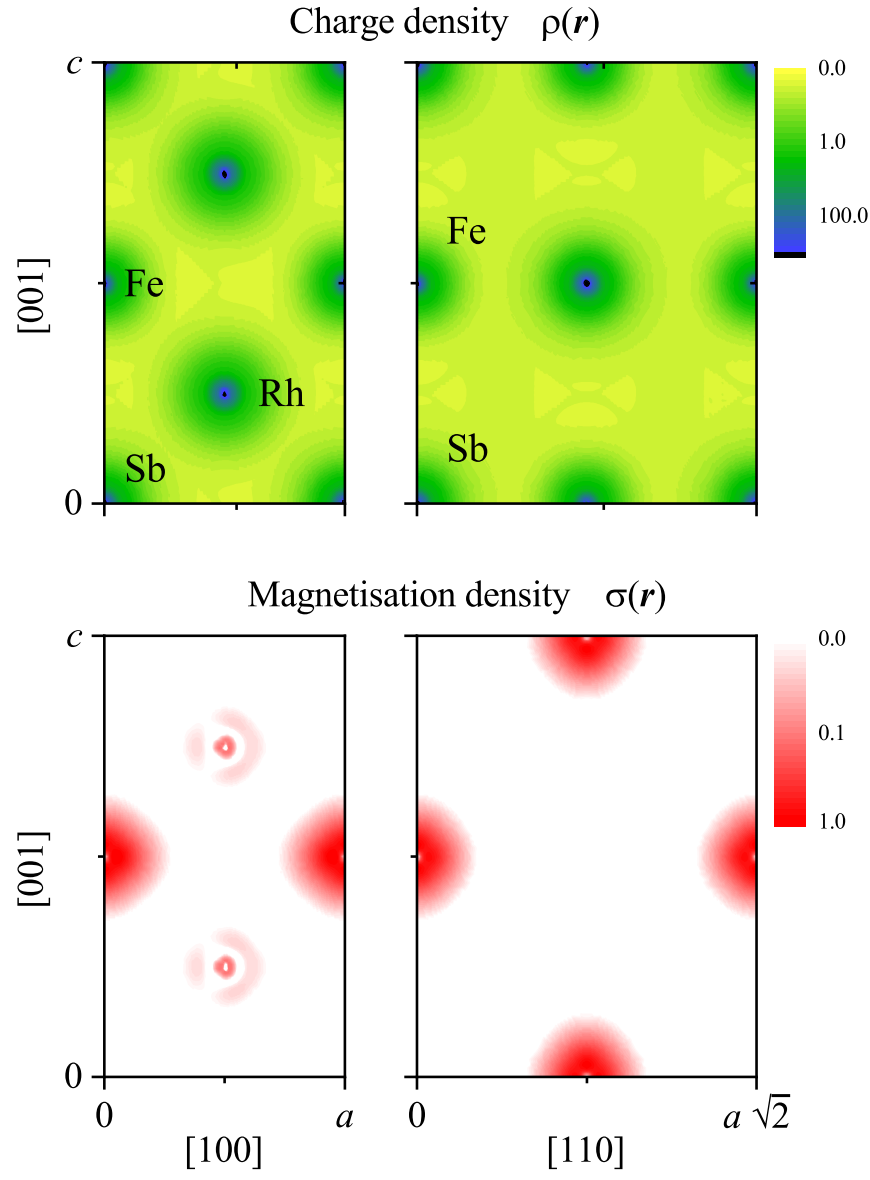

FIG. 5. Electronic structure of $\mathrm{Rh}_{2} \mathrm{FeSb}$ (II). Fully relativistic charge $[\rho(r)]$ and spin $[\sigma(r)]$ distributions for different planes. Magnetization is perpendicular to $c$ with $m$ along [100] in accordance with the easy plane behavior of the magnetic anisotropy. (Note: Color bars are in atomic units.)

TABLE III. Calculated magnetic properties of $\mathrm{Rh}_{2} \mathrm{FeSb}$, $\mathrm{Rh}_{2} \mathrm{Fe}_{0.5} \mathrm{Co}_{0.5} \mathrm{Sb}$, and $\mathrm{Rh}_{2} \mathrm{CoSb}$. Spin $m_{s}$ and orbital $m_{l}$ magnetic moments per atom (Rh, $T=\mathrm{Co}$, Fe with $m \| c$ in all cases) of the primitive cell (total) are listed, as well as Curie temperature $T_{C}$, spin stiffness $D_{0}$, and anisotropy parameters. (Note that the dipolar anisotropy is three orders of magnitude lower than the magnetocrystalline part.)

\begin{tabular}{lccc}
\hline \hline & $\mathrm{Fe}$ & $\mathrm{Fe}_{0.5} \mathrm{Co}_{0.5}$ & $\mathrm{Co}$ \\
\hline$m_{s}^{\mathrm{Rh}}\left(\mu_{B}\right)$ & 0.237 & 0.239 & 0.204 \\
$m_{l}^{\mathrm{Rh}}\left(\mu_{B}\right)$ & 0.006 & 0.008 & 0.006 \\
$m_{s}^{\mathrm{Fe}}\left(\mu_{B}\right)$ & 3.006 & 2.977 & - \\
$m_{l}^{\mathrm{Fe}}\left(\mu_{B}\right)$ & 0.080 & 0.084 & - \\
$m_{s}^{\mathrm{Co}}\left(\mu_{B}\right)$ & - & 1.747 & 1.674 \\
$m_{l}^{\mathrm{Co}}\left(\mu_{B}\right)$ & - & 0.132 & 0.137 \\
$m_{s}^{\text {tot }}\left(\mu_{B}\right)$ & 3.44 & 2.81 & 2.04 \\
$m_{l}^{\text {tot }}\left(\mu_{B}\right)$ & 0.09 & 0.12 & 0.15 \\
$T_{C}(\mathrm{~K})$ & 465 & 480 & 500 \\
$D_{0}\left(\mathrm{meV} \AA^{2}\right)$ & 590 & 700 & 870 \\
$K_{u}\left(\mathrm{MJ} / \mathrm{m}^{3}\right)$ & -1.21 & -0.23 & 1.37 \\
$\left|\mu_{0} H_{a}\right|(\mathrm{T})$ & 1.34 & 0.31 & 2.43 \\
$\Delta E_{\text {dipaniso }}\left(\mathrm{kJ} / \mathrm{m}^{3}\right)$ & 1.9 & & 2.0 \\
\hline \hline
\end{tabular}




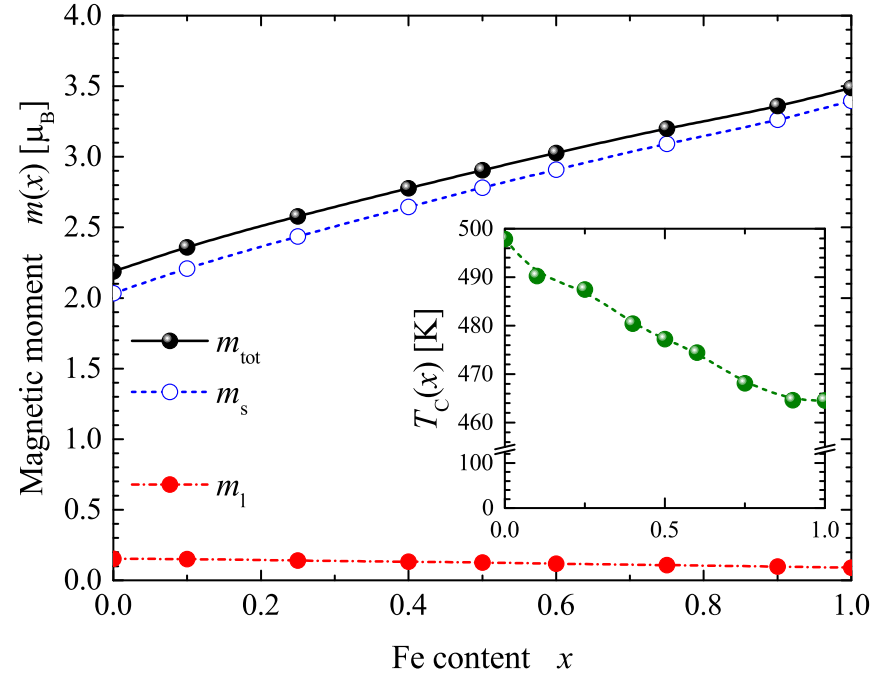

FIG. 6. Magnetic properties of $\mathrm{Rh}_{2} \mathrm{Fe}_{x} \mathrm{Co}_{1-x} \mathrm{Sb}$. Total $\left(m_{\mathrm{tot}}\right)$, spin $\left(m_{s}\right)$, and orbital $\left(m_{l}\right)$ magnetic moments as functions of Fe content $x$ are shown. The inset shows the Curie temperature $\left(T_{C}\right)$.

The anisotropy has the hard axis along the $z$ ([001]) direction, and the easy plane is the the basal plane. By contrast, for $\mathrm{Rh}_{2} \mathrm{CoSb}$, the $z$ direction is the easy axis. The simple uniaxial anisotropy constant is $K_{u}=-1.21 \mathrm{MJ} / \mathrm{m}^{3}$. Consequently, the anisotropy field is $\left|\mu_{0} H_{a}\right|=1.34 \mathrm{~T}$. The appearance of the hard axis along $z$ is opposite to $\mathrm{Rh}_{2} \mathrm{CoSb}$ where $z$ is the easy axis. The dipolar magnetocrystalline anisotropy of $\mathrm{Rh}_{2} \mathrm{FeSb}$ is $\Delta E_{\text {dipaniso }}=0.09 \mu \mathrm{eV}$, indicating that the easy dipolar direction is along the [001] axis, like that of the Co-containing compound. This behavior is caused by the strong magnetic moments of the $3 d$ transition metals, in addition to the elongation of the tetragonal crystal structure along the $c$ axis.

\section{Results for $\mathrm{Rh}_{2} \mathrm{Fe}_{x} \mathrm{Co}_{1-x} \mathrm{Sb}$}

Owing to the differences in magnetic anisotropy between the $\mathrm{Fe}$ - and Co-based compounds, it is interesting to investigate a mixed system containing both $\mathrm{Fe}$ and $\mathrm{Co}$. Therefore, calculations were also performed for $\mathrm{Rh}_{2} \mathrm{Fe}_{x} \mathrm{Co}_{1-x} \mathrm{Sb}$ using SPRKKR and the CPA. The CPA enables the simulation of random occupation of $\mathrm{Fe}$ and $\mathrm{Co}$ atoms at a single site (here $2 b$ ). In the calculations, lattice parameters were interpolated from those of the pure compounds using Veegard's law. For $\mathrm{Rh}_{2} \mathrm{Fe}_{0.5} \mathrm{Co}_{0.5} \mathrm{Sb}$, they are close to the experimental values measured for a twinned single crystal ( $a=4.0487 \AA, c=$ $7.1011 \AA$ ). For this composition, a formation enthalpy of $\Delta H_{f}=-414 \mathrm{meV}$ was found. The obtained magnetic properties of $\mathrm{Rh}_{2} \mathrm{Fe}_{0.5} \mathrm{Co}_{0.5} \mathrm{Sb}$ are shown in Table III in comparison with the pure compounds. The uniaxial anisotropy constant is negative, like that of $\mathrm{Rh}_{2} \mathrm{FeSb}$; however, its absolute value is considerably lower (by a factor of 35) than that of $\mathrm{Rh}_{2} \mathrm{CoSb}$.

The dependence of the magnetic properties on the composition is shown in Fig. 6. The total magnetic moment increases with increasing Fe content, mainly because Fe has a higher spin magnetic moment $\left(\approx 3 \mu_{B}\right)$ than $\mathrm{Co}\left(\approx 1.7 \mu_{B}\right)$. The individual magnetic moments of the atoms are nearly unaffected by the composition. The calculated Curie temperature decreases with increasing Fe content.

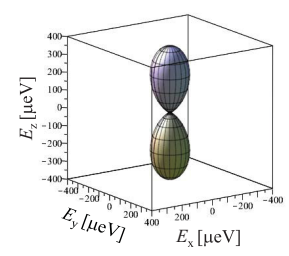

(a) $\mathrm{Rh}_{2} \mathrm{FeSb}$

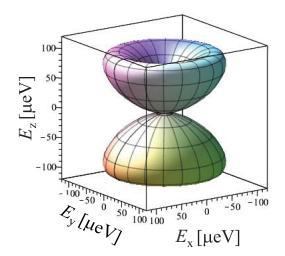

(b) $\mathrm{Rh}_{2} \mathrm{Fe}_{0.5} \mathrm{Co}_{0.5} \mathrm{Sb}$

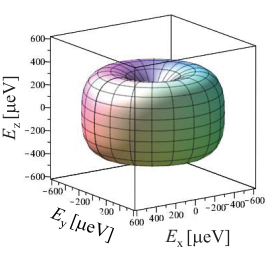

(c) $\mathrm{Rh}_{2} \mathrm{CoSb}$
FIG. 7. Uniaxial magnetic anisotropy of $\mathrm{Rh}_{2} T \mathrm{Sb}$ compounds. Energy distributions $E_{u^{\prime}}(\theta, \phi)$ of $T=\mathrm{Fe}$ (a), $\mathrm{Fe}_{0.5} \mathrm{Co}_{0.5}$ (b), and $\mathrm{Co}$ (c). (Please note the different energy scales.)

\section{Magnetocrystalline anisotropy of $\mathrm{Rh}_{2} \mathrm{Fe}_{x} \mathrm{Co}_{1-x} \mathrm{Sb}$}

Thus far, only the simplest case of uniaxial magnetocrystalline anisotropy has been considered. The equations for extending the calculations to more detailed cases are given in Appendix D. These equations were used to calculate the fourth-order uniaxial and tetragonal anisotropy constants, which were used to obtain the magnetocrystalline anisotropy energy distributions.

The calculated uniaxial energy distributions $E_{u^{\prime}}(\theta, \phi)$ [see Eqs. (D4) and (D18) in the Appendix] of $\mathrm{Rh}_{2} \mathrm{FeSb}$, $\mathrm{Rh}_{2} \mathrm{Fe}_{0.5} \mathrm{Co}_{0.5} \mathrm{Sb}$, and $\mathrm{Rh}_{2} \mathrm{CoSb}$ are plotted in Fig. 7 for comparison.

The different behavior of the anisotropy is clearly revealed in Fig. 7. $\mathrm{Rh}_{2} \mathrm{FeSb}$ has an easy plane, and $c$ is the hard axis; $\mathrm{Rh}_{2} \mathrm{Fe}_{0.5} \mathrm{Co}_{0.5} \mathrm{Sb}$ has an easy plane as well, but a hard cone, and in $\mathrm{Rh}_{2} \mathrm{CoSb}$, the $c$ direction is the easy axis. $\mathrm{Rh}_{2} \mathrm{Fe}_{0.5} \mathrm{Co}_{0.5} \mathrm{Sb}$ has a much lower anisotropy than the pure compounds, and the differences between the energies of the $a b$ plane and the $c$ axis are very small. A hard cone appears with its maximum at an angle of $\theta_{3,4}= \pm 35.7^{\circ}$ [see Eq. (D9) in Appendix D1].

The calculated anisotropy constants for uniaxial and tetragonal symmetry are compared in Table IV. The simple $K_{u}$ from Eq. (D2) (see Appendix D) clearly cannot describe the magnetic anisotropy correctly.

The dependence of the uniaxial anisotropy constants on the composition is illustrated in Fig. 8. The uniaxial anisotropy constant $K_{u}$ decreases with increasing iron content and exhibits a zero-crossing at $x_{0} \approx 0.4$. At intermediate iron contents, more complex behavior appears, as shown by the

TABLE IV. Comparison of the anisotropy constants of $\mathrm{Rh}_{2} T \mathrm{Sb}$, $T=\mathrm{Fe}, \mathrm{Fe}_{0.5} \mathrm{Co}_{0.5}$, and $\mathrm{Co}$.

\begin{tabular}{lrcc}
\hline \hline & $\mathrm{Rh}_{2} \mathrm{FeSb}$ & $\mathrm{Rh}_{2} \mathrm{Fe}_{0.5} \mathrm{Co}_{0.5} \mathrm{Sb}$ & $\mathrm{Rh}_{2} \mathrm{CoSb}$ \\
\hline uniaxial & & & \\
$K_{u}\left(\mathrm{MJ} / \mathrm{m}^{3}\right)$ & -1.21 & -0.23 & 1.37 \\
$K_{0}\left(\mathrm{MJ} / \mathrm{m}^{3}\right)$ & 1.31 & 0.39 & 0.0 \\
$K_{2}\left(\mathrm{MJ} / \mathrm{m}^{3}\right)$ & -2.19 & 0.50 & 3.62 \\
$K_{4}\left(\mathrm{MJ} / \mathrm{m}^{3}\right)$ & 0.98 & -0.73 & -2.25 \\
tetragonal & & & \\
$K_{0,0}\left(\mathrm{MJ} / \mathrm{m}^{3}\right)$ & 1.31 & 0.39 & 0.0 \\
$K_{2,0}\left(\mathrm{MJ} / \mathrm{m}^{3}\right)$ & -2.19 & 0.50 & 3.62 \\
$K_{4,0}\left(\mathrm{MJ} / \mathrm{m}^{3}\right)$ & 0.93 & -0.81 & -2.40 \\
$K_{4,4}\left(\mathrm{MJ} / \mathrm{m}^{3}\right)$ & 0.05 & 0.08 & 0.15 \\
\hline \hline
\end{tabular}




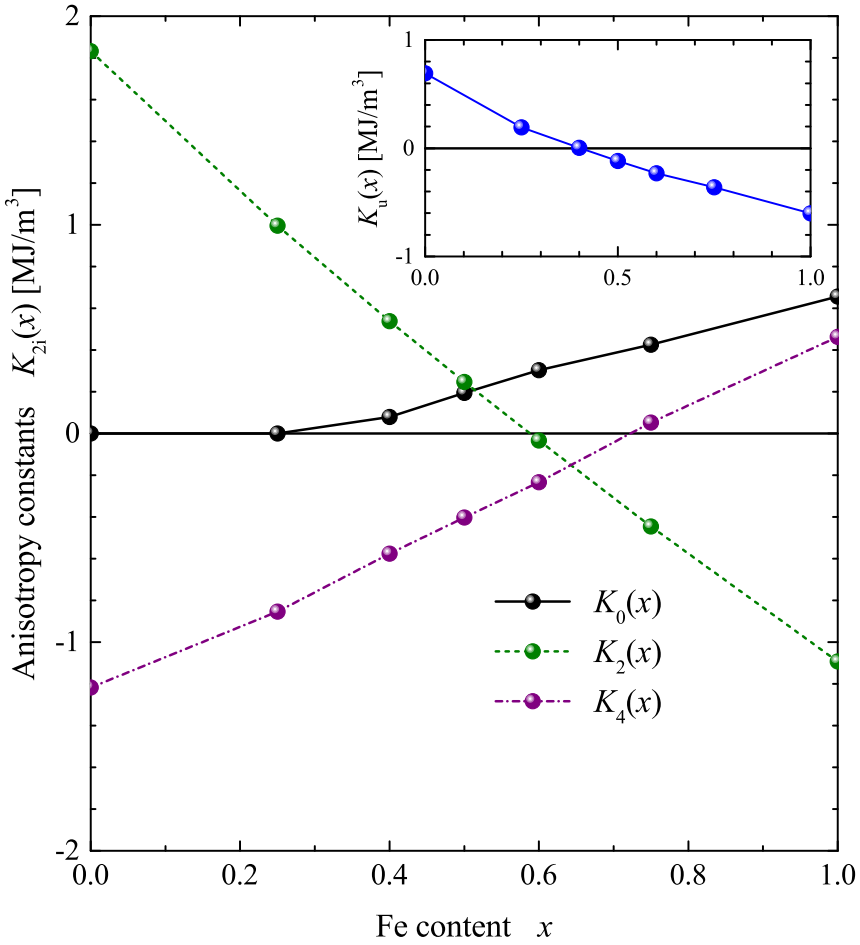

FIG. 8. Anisotropy constants of $\mathrm{Rh}_{2} \mathrm{Fe} x \mathrm{Co}_{1-x} \mathrm{Sb}$ compounds. The inset shows the uniaxial anisotropy constant obtained using Eq. (D3) in Appendix D1.

composition dependence of $K_{2 i}$ and the results in Figs. 7 and 9.

The calculated tetragonal energy distributions $E_{a^{\prime}}(\theta, \phi)$ [see Eqs. (D12) and (D18) in the Appendixes] of $\mathrm{Rh}_{2} \mathrm{FeSb}$, $\mathrm{Rh}_{2} \mathrm{Fe}_{0.5} \mathrm{Co}_{0.5} \mathrm{Sb}$, and $\mathrm{Rh}_{2} \mathrm{CoSb}$ are shown in Fig. 9. As in the plot of the uniaxial anisotropy in Fig. 7, the differences in the anisotropy are easily observed. In $\mathrm{Rh}_{2} \mathrm{FeSb}$, the hard axis is along the $z$ ([001]) direction, and the anisotropy exhibits weak variation in the basal plane, which is close to the easy plane. Closer examination of the basal plane shows biaxial behavior with easy axes along the [110] and [11̄0] axes, but the energy difference between these directions and the [100] or [010] axes is very small. The anisotropy of $\mathrm{Rh}_{2} \mathrm{CoSb}$ is still almost uniaxial, with the easy axis along the $c$ ([001]) axis, and varies weakly in the basal plane. $\mathrm{Rh}_{2} \mathrm{Fe}_{0.5} \mathrm{Co}_{0.5} \mathrm{Sb}$ has much lower anisotropy than the pure compounds and exhibits more complicated directional behavior.

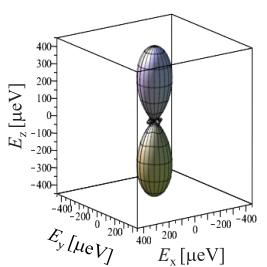

(a) $\mathrm{Rh}_{2} \mathrm{FeSb}$

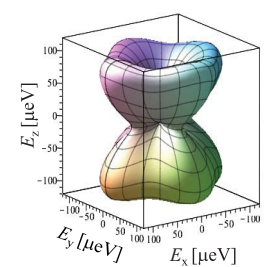

(b) $\mathrm{Rh}_{2} \mathrm{Fe}_{0.5} \mathrm{Co}_{0.5} \mathrm{Sb}$

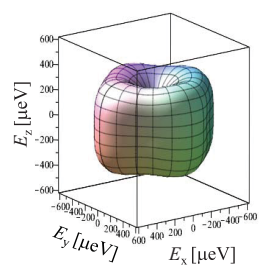

(c) $\mathrm{Rh}_{2} \mathrm{CoSb}$
FIG. 9. Tetragonal magnetic anisotropy of $\mathrm{Rh}_{2} T \mathrm{Sb}$ compounds. Energy distributions $E_{a^{\prime}}(\theta, \phi)$ of $T=\mathrm{Fe}(\mathrm{a}), \mathrm{Fe}_{0.5} \mathrm{Co}_{0.5}$ (b), and $\mathrm{Co}$ (c). (Please note the different energy scales.)

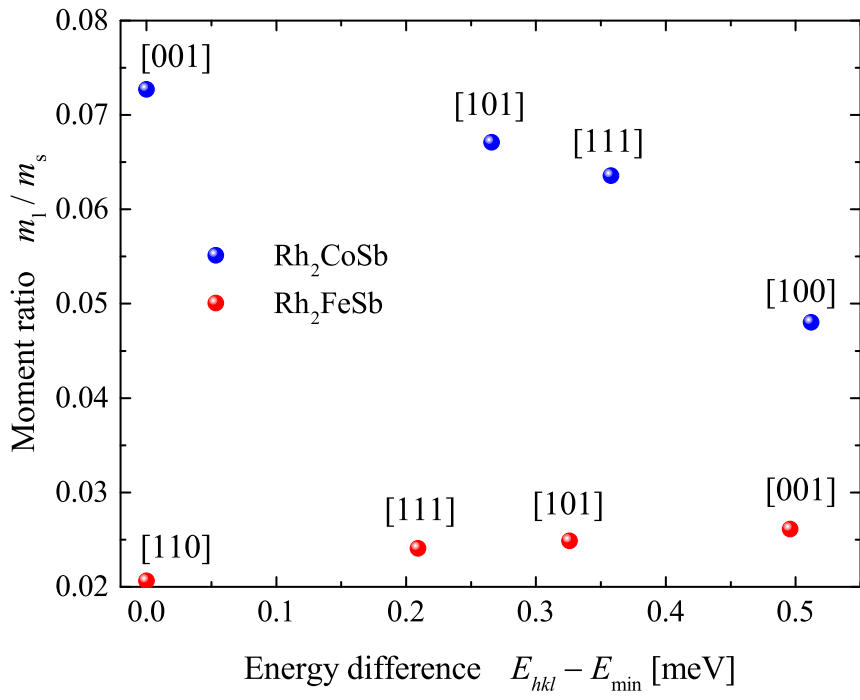

FIG. 10. Directional dependence of the orbital moments of $\mathrm{Rh}_{2} T^{\prime} \mathrm{Sb}, T^{\prime}=\mathrm{Co}, \mathrm{Fe}$. Note that the orbital moments are given relative to the spin moments for better comparison.

The directional dependence of the orbital magnetic moments was analysed to clarify the role of the spin-orbit interaction. The magnetic moments for $m \| c$ are listed in Table III. The ratio of the total orbital moment to the total spin moment, $m_{l} / m_{s}$, was used owing to the large differences between the magnetic moments for different compositions. Figure 10 shows the ratio $m_{l} / m_{s}$ as a function of the difference in the energies in several magnetization directions $[h k l]$. For both $\mathrm{Rh}_{2} \mathrm{CoSb}$ and $\mathrm{Rh}_{2} \mathrm{FeSb}$, the ratio is largest for magnetization along the $c$ axis ([001]) and lowest in the basal plane. This finding involves not only the ratio but also the orbital momenta themselves, indicating that the orbital moment is not always largest when the magnetization is along the easy axis (or in the easy plane). Here it depends at least partially on the angle between the magnetization and $c$ axis, as shown by the values for other directions.

To further examine the nature of the anisotropy, the charge and spin density distributions were analyzed with respect to the magnetization direction (compare also Figs. 3 and 5). As mentioned above, the symmetry changes when the magnetization is applied along different crystallographic directions. The point group symmetry of the $2 b$ sites occupied by $\mathrm{Fe}$ and Co is $D_{4 h}$ and $D_{2 d}$ for $\mathrm{Rh}$ on $4 d$. Applying the magnetization along one of the high-symmetry axes, i.e., the $c$ ([001]) or $a$ ([100]) axis, changes the symmetry of the $2 b$ sites to $C_{4 h}$ or $C_{2 h}$, respectively. As a result, the irreducible representations and basic functions depend on the magnetization direction. For $C_{4 h}$, they are $a_{g}, b_{g}$, and $e_{g}$ with the $l=2$ basic functions $d_{z^{2}},\left(d_{x^{2}-y^{2}}, d_{x y}\right)$, and $\left(d_{x z}, d_{y z}\right)$. For $C_{2 h}$, they are $a_{g}$ and $b_{g}$ with $\left(d_{z^{2}}, d_{x^{2}-y^{2}}, d_{x y}\right)$ and $\left(d_{x z}, d_{y z}\right)$. Similar differences appear for the $4 d$ sites. The charge and spin density distributions for different magnetization directions are compared in Fig. 11 for the compounds containing only Fe or Co.

As mentioned above, the details of the charge density are not easily observed directly from the graph when the magnetization direction is changed because the graph mainly shows the positions of the atoms where the charge is always highest. 

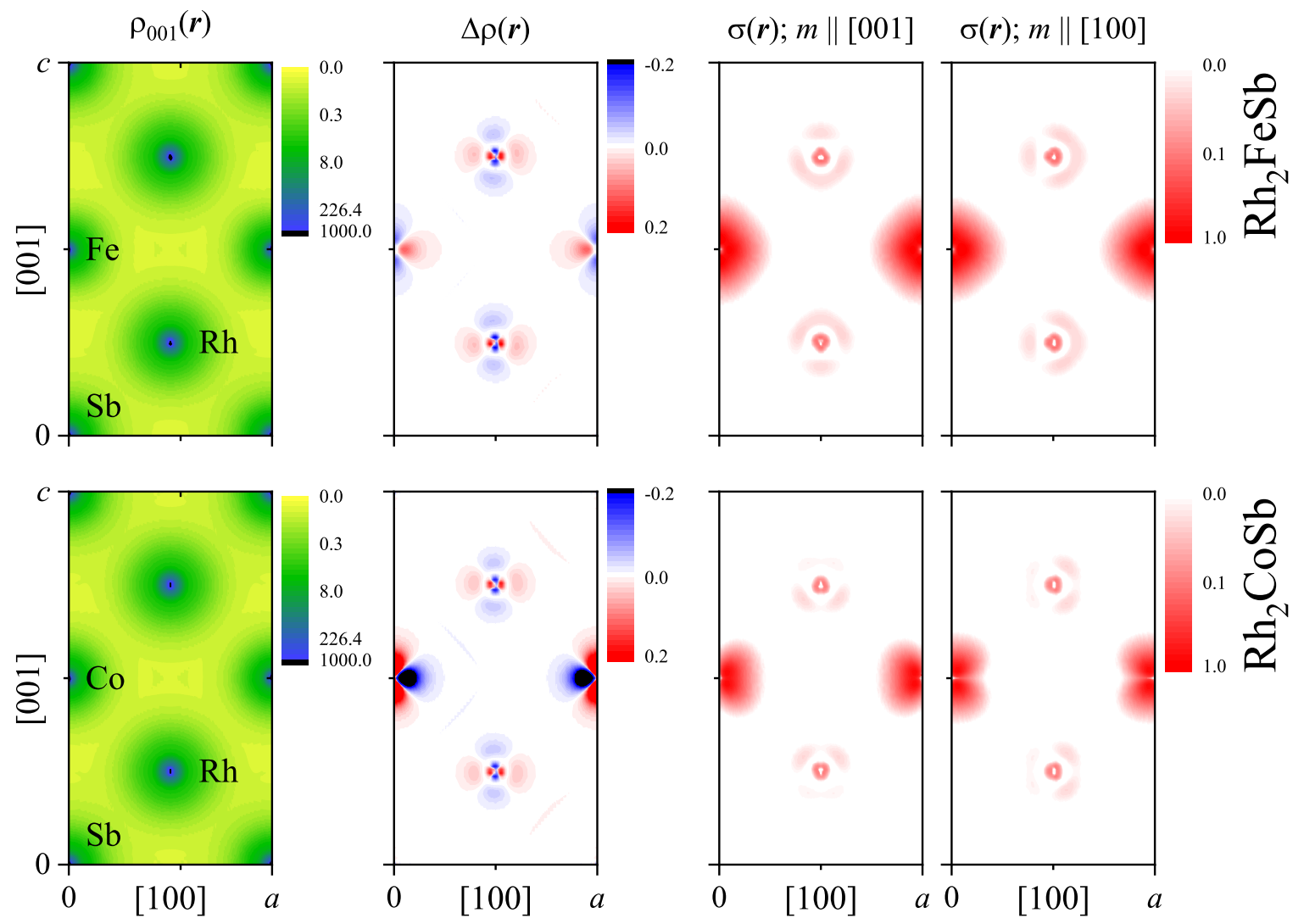

FIG. 11. Electronic structure of $\mathrm{Rh}_{2} \mathrm{FeSb}$ and $\mathrm{Rh}_{2} \mathrm{CoSb}$. Fully relativistic charge $[\rho(r)]$ and spin $[\sigma(r)]$ density distributions in a (100)-type plane for magnetization parallel and perpendicular to the $c$ axis are shown. $\rho_{001}(r)$ is the charge density for $m \|[001]$ and $\Delta \rho=\rho_{001}-\rho_{100}$ is the difference between the charge densities for $m \|[001]$ and $m \|[100]$. (Note: Color bars are in atomic units.)

However, they can be observed if one investigates the difference in the charge distribution, which is plotted as $\Delta \rho(r)$. It was calculated for both compounds as the difference between the charge densities obtained, assuming that the magnetization is parallel $(m \|[001])$ or perpendicular $(m \|[100])$ to the $c$ axis. In both compounds, the magnetization has the same effect on $\Delta \rho(r)$ near the Rh atoms. That is, the charge distribution is rotated with the direction of the magnetization. In the same way, the Rh-based spin densities are affected by the magnetization direction. They change from [001] aligned when $m \|[001]$ to [100] aligned when $m \|[100]$, regardless of which $3 d$ transition metal is used. The situation is different near the $3 d$ transition metals $\mathrm{Fe}$ and $\mathrm{Co}$, where $\Delta \rho(r)$ and $\sigma(r)$ are affected very differently by the magnetization direction. The reason is the different occupation of $3 d$ valence electrons of $\mathrm{Fe}$ $\left(n_{d}^{\mathrm{Fe}}=6.6\right)$ and Co $\left(n_{d}^{\mathrm{Co}}=7.8\right)$, which are responsible for the different spin moments. The overall differences in the charge and spin densities at different magnetization directions result in different total energies.

Finally, the gain or loss of energy with changes in the magnetization direction results in the magnetocrystalline anisotropy. The electronic structure of the two compounds, $\mathrm{Rh}_{2} \mathrm{FeSb}$ and $\mathrm{Rh}_{2} \mathrm{CoSb}$, differs depending on the magnetization direction, which is reflected in the change in the anisotropy from the easy plane to the easy axis when $\mathrm{Fe}$ is replaced with Co.

\section{CONCLUSIONS}

The electronic and magnetic structure of tetragonal Heusler compounds with the composition $\mathrm{Rh}_{2} \mathrm{Fe}_{x} \mathrm{Co}_{1-x} \mathrm{Sb}$ were investigated by $a b$ initio calculations. The calculations revealed that the magnetic moment increases and the Curie temperature decreases with increasing $\mathrm{Fe}$ content $x$. The $\mathrm{Rh}$ atoms have only small, composition-independent magnetic moments. The magnetic properties are determined by those of the $\mathrm{Fe}$ and Co atoms and thus depend strongly on the composition. The total energies for various magnetization directions were calculated to determine the magnetic anisotropy. The analysis is described in detail in the extended Appendixes. For bulk materials, the magnetocrystalline anisotropy is found to be much stronger (by three orders of magnitude) than the dipolar anisotropy. Special attention was given to the borderline compounds, $\mathrm{Rh}_{2} \mathrm{FeSb}$ and $\mathrm{Rh}_{2} \mathrm{CoSb}$. The most striking result was that a composition-dependent transition from easy-axis to easy-plane anisotropy occurs at an iron concentration of approximately $40 \%$.

\section{ACKNOWLEDGMENTS}

We thank the groups of P. Blaha (Vienna) and H. Ebert (Munich) for providing their computer codes. 


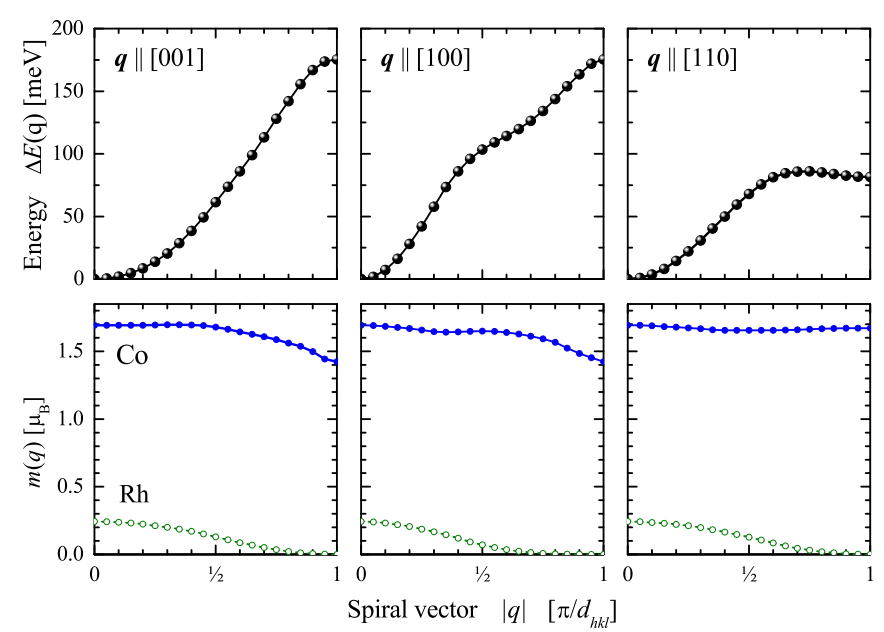

FIG. 12. Planar spin spirals in $\mathrm{Rh}_{2} \mathrm{CoSb}$. The spiral energies are given with respect to $q=0$, that is, $\Delta E(q)=E(q)-E(0)$. The behavior of the magnetic moment $m(q)$ is shown for Rh and Co.

\section{APPENDIX A: DYNAMIC, MAGNETIC PROPERTIES}

Information about the dynamic, magnetic properties is important. Spin spirals, exchange coupling coefficients, and magnons are related to each other and become manifest in the Curie temperature, for example. They were investigated for the pure compounds. Here, the magnons do not show any instabilities. The absence of soft modes or complex (imaginary) energies confirms that the assumed magnetic structures are stable. This is similar to the fact that phonon instabilities in calculations would point to instability of the assumed geometrical structure.

\section{Spiral spin order in $R_{2} \mathbf{C o S b}$}

The energy of the spin spirals was calculated to search for non-collinear magnetic order. The spin spirals were calculated for different directions and different cones. In planar spirals, the spins are perpendicular to the propagation direction. Figure 12 compares the energies of planar spirals along the high-symmetry directions.

The spirals along [100] or [110] propagate in the fourfold plane, whereas the spiral in the [001] direction propagates along the $c$ axis. In all cases, the lowest energy is observed at $q=0$. The magnetic moment of the Co atoms varies by approximately $17 \%$ at maximum. The magnetic moment of the $\mathrm{Rh}$ atoms decreases with increasing $q$ and vanishes at the border, independent of the propagation direction of the spiral.

The spin direction was assumed to be perpendicular to the $q$ vector in the above calculations for planar spirals. Thus, the angle between $\vec{q}$ and the local magnetic moment $\vec{m}_{i}$ was set to $\Theta=\pi / 2$. Next, the spirals were assumed to be conical with $0<\Theta<\pi / 2$ to allow for a more detailed analysis. The calculations were performed for $q$ along [001]. Figure 13 displays the results for conical spirals with various cone angles. The highest energies appear for the planar spiral. The energy at the border of the Brillouin zone $(q=\pi / c)$ exhibits a sine dependence. Thus, it vanishes in the antiferromagnetic state. The behavior of the local magnetic moments suggests more

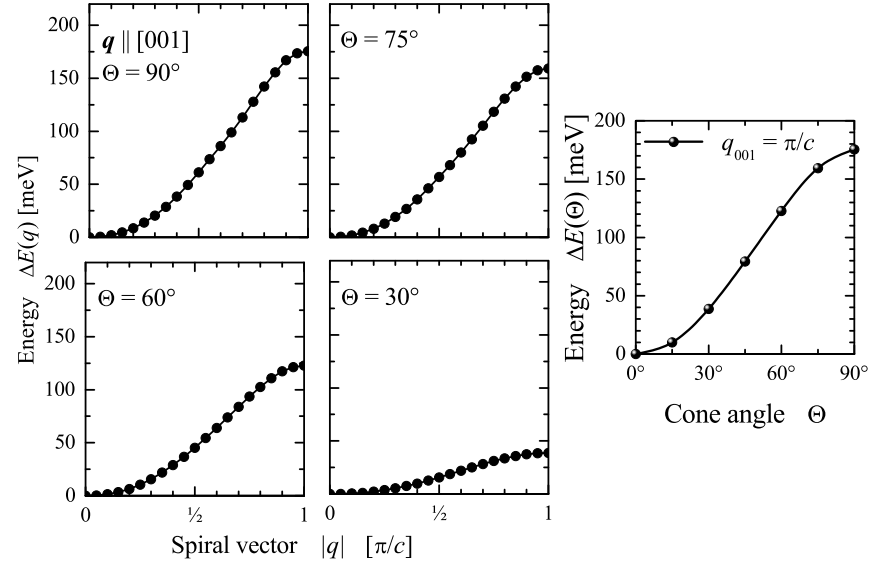

FIG. 13. Conical spirals in $\mathrm{Rh}_{2} \mathrm{CoSb}$. Spiral energies for different cone angles and the wave vector along the $c$ axis $(q \|[001])$ are shown. The angular dependence at $q=\pi / c$ is also shown.

localised behavior at the Co atoms and induced behavior at the $\mathrm{Rh}$ atoms.

The calculated spiral energies indicate that this type of magnetic order is rather improbable. The spiral energies increase monotonously with the wave vector and cone angle, rather independent on the $\vec{q}$ direction. The monotonic behavior suggests that a canted magnetic order is also very unlikely [19].

\section{Exchange coupling and magnons in $\mathrm{Rh}_{2} \mathrm{CoSb}$}

The exchange coupling energies were calculated using the scheme of Liechtenstein et al. [20,21] to estimate the Curie temperature, spin stiffness, and presence of magnons [14]. The exchange coupling parameters are plotted in Figure 14(a). The most dominant parameters for $\mathrm{Co}-\mathrm{Co}$ and $\mathrm{Co}-\mathrm{Rh}$ interactions are shown; all the others are comparatively small. The largest interaction appears for Co atoms in the center and nearest to the $\mathrm{Co}$ in the neighboring plane. From the calculated exchange coupling energies, the Curie temperature was found to be $T_{C}=498 \mathrm{~K}$, which is close to the experimental value $(450 \mathrm{~K})$ [1]. The calculated spin-wave stiffness constant is $D_{i j}=866 \mathrm{meV} \AA^{2}$, and the interpolation scheme of Padja et al. [22] yields an extrapolated spin wave stiffness of $D_{0}=864 \mathrm{meV} \AA^{2}$.

The magnon dispersion was calculated by Fourier transformation of the real-space exchange coupling parameters. The result is presented in Fig. 14(b) and the magnon density of states is shown in Fig. 14(c). Two calculations were made; in one calculation, only the Co-Co interaction was considered, and in the other, the moments of the $\mathrm{Rh}$ atoms, which result in additional $\mathrm{Co}-\mathrm{Rh}$ and $\mathrm{Rh}-\mathrm{Rh}$ coupling, were included. The latter calculation yields flat dispersion curves and a high density of states. A comparison of the two calculations reveals that the magnons are dominated by the Co-Co interaction. Note that the Curie temperature in only $10 \mathrm{~K}$ lower when the $\mathrm{Rh}$ moments and the corresponding exchange parameters are ignored. 

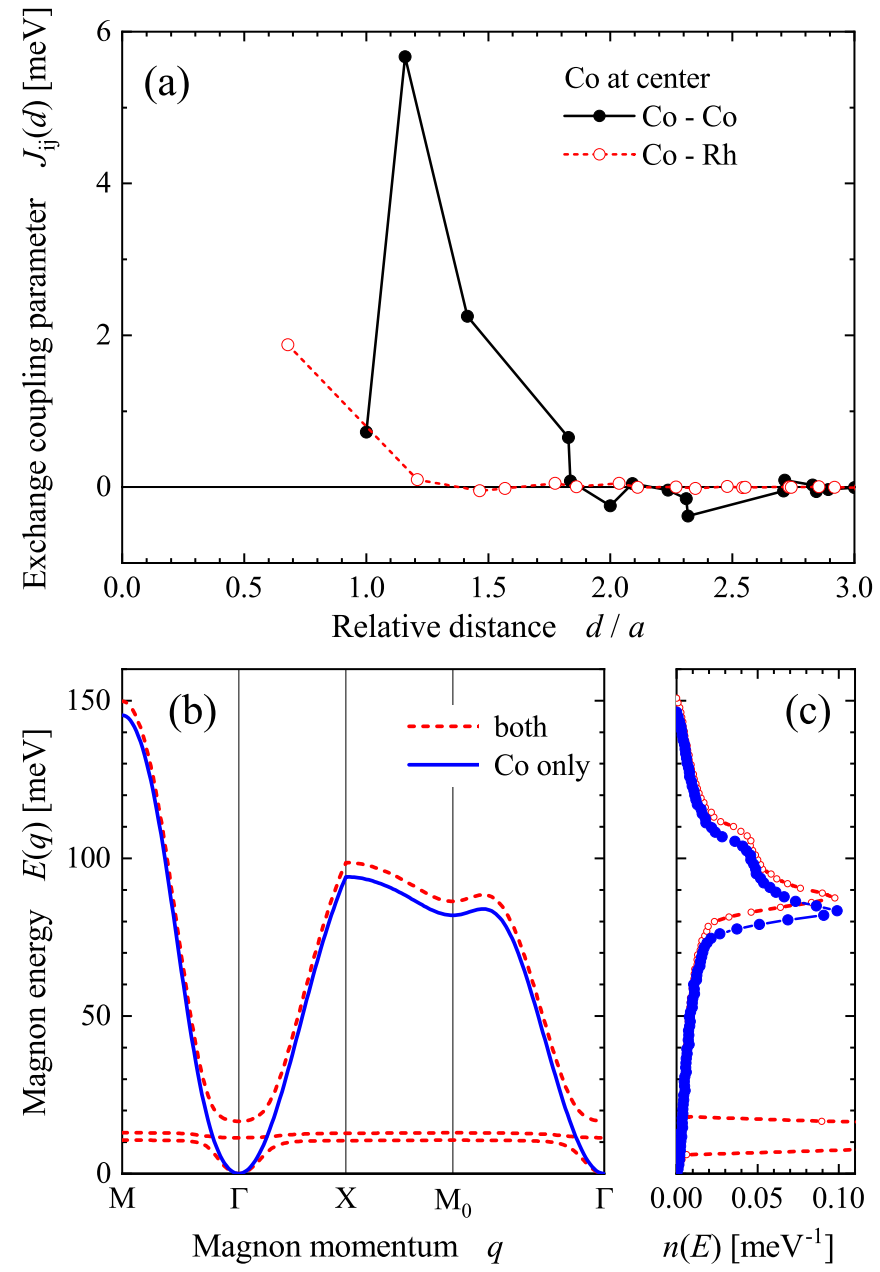

FIG. 14. Exchange coupling parameters of $\mathrm{Rh}_{2} \mathrm{CoSb}$. (a) Exchange coupling parameters for $\mathrm{Co}-\mathrm{Co}$ and $\mathrm{Co}-\mathrm{Rh}$ interaction as functions of distance. Lines are drawn for better comparison. (b) Magnon dispersion and (c) density of states. Calculations were performed with and without accounting for the magnetic moment of the $\mathrm{Rh}$ atoms (that is, for both atoms and for Co only).

\section{Spin spirals and magnons in $\mathrm{Rh}_{2} \mathrm{FeSb}$}

The dynamic magnetic properties of $\mathrm{Rh}_{2} \mathrm{FeSb}$ are shown in Fig. 15. The spin spirals and magnons are similar to those of $\mathrm{Rh}_{2} \mathrm{CoSb}$; however, their energies extend to higher values. The behavior of the spin spirals rules out the presence of noncollinear magnetic structure [19].

\section{APPENDIX B: DISORDER}

\section{1. $\mathrm{Rh}_{2} \mathrm{CoSb}$ with $\mathrm{Co}-\mathrm{Sb}$-type antisite disorder}

Supplementary calculations were performed for disordered $\mathrm{Rh}_{2} \mathrm{CoSb}$ and $\mathrm{Rh}_{2} \mathrm{FeSb}$ with SPRKKR using the CPA. For example, the disordered compound may be written as $\mathrm{Rh}_{2}\left(\mathrm{Co}_{1-x / 2} \mathrm{Sb}_{x / 2}\right)\left(\mathrm{Co}_{x / 2} \mathrm{Sb}_{1-x / 2}\right)$, where $x$ is the disorder level. The result for $x=1$, which denotes complete $\mathrm{Co}-\mathrm{Sb}$ disorder, is illustrated in Fig. 1(b). Alternatively, it can be assumed that disorder between the $\mathrm{Co}$ and $\mathrm{Rh}$ atoms decreases the magnetic moments, which is consistent with the results of calculations of the inverted structure in space group No. 119,
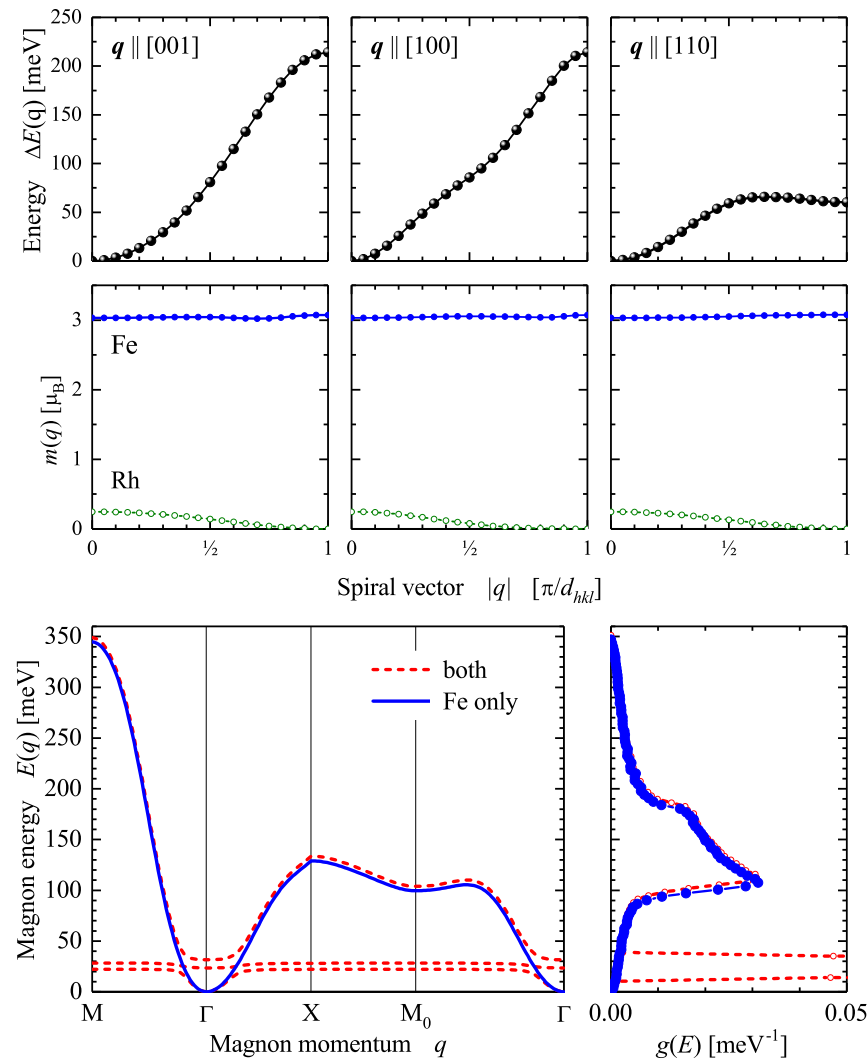

FIG. 15. Dynamic magnetic properties of $\mathrm{Rh}_{2} \mathrm{FeSb}$. Spiral energies with corresponding magnetic moments and the magnon dispersion are shown, along with the magnon density of states $g(E)$. Magnon calculations were performed with and without the magnetic moment of the $\mathrm{Rh}$ atoms; in the latter case, all $\mathrm{Fe}-\mathrm{Rh}$ interactions are neglected.

but not with those when $\mathrm{Co}-\mathrm{Sb}$ disorder is assumed, as shown below.

The evolution of the magnetic moments of $\mathrm{Rh}_{2} \mathrm{CoSb}$ and $\mathrm{Rh}_{2} \mathrm{FeSb}$ with increasing disorder is shown in Fig. 16. The total magnetic moment in the fully disordered state is approximately $20 \%$ larger for $\mathrm{Rh}_{2} \mathrm{CoSb}$ and approximately $10 \%$ larger for $\mathrm{Rh}_{2} \mathrm{FeSb}$ than those of the compounds in the completely ordered state. The orbital moments are nearly constant in both compounds and are independent of the degree of disorder $(x)$. The decrease in the total moments with decreasing $x$ is attributed to the decrease in the spin magnetic moments of both compounds.

Figure 16 shows that the type of anisotropy (easy axis for $\mathrm{Rh}_{2} \mathrm{CoSb}$ and easy plane for $\mathrm{Rh}_{2} \mathrm{FeSb}$ ) is retained even in the completely disordered state. However, the absolute value of the second-order uniaxial anisotropy constant $K_{u}$ decreases. That is, the anisotropy becomes weaker with increasing disorder. The $K_{u}$ values of both compounds are approximately $70 \%$ higher in the completely disordered state. No direct correspondence is observed between the behavior of $K_{u}$ and that of the spin, orbital, or total magnetic moments. The effects of disorder and composition on the magnetic anisotropy of the Co-Fe system were investigated using first-principles CPA calculations by Turek et al. [23], who also observed a decrease in anisotropy with increasing disorder. 

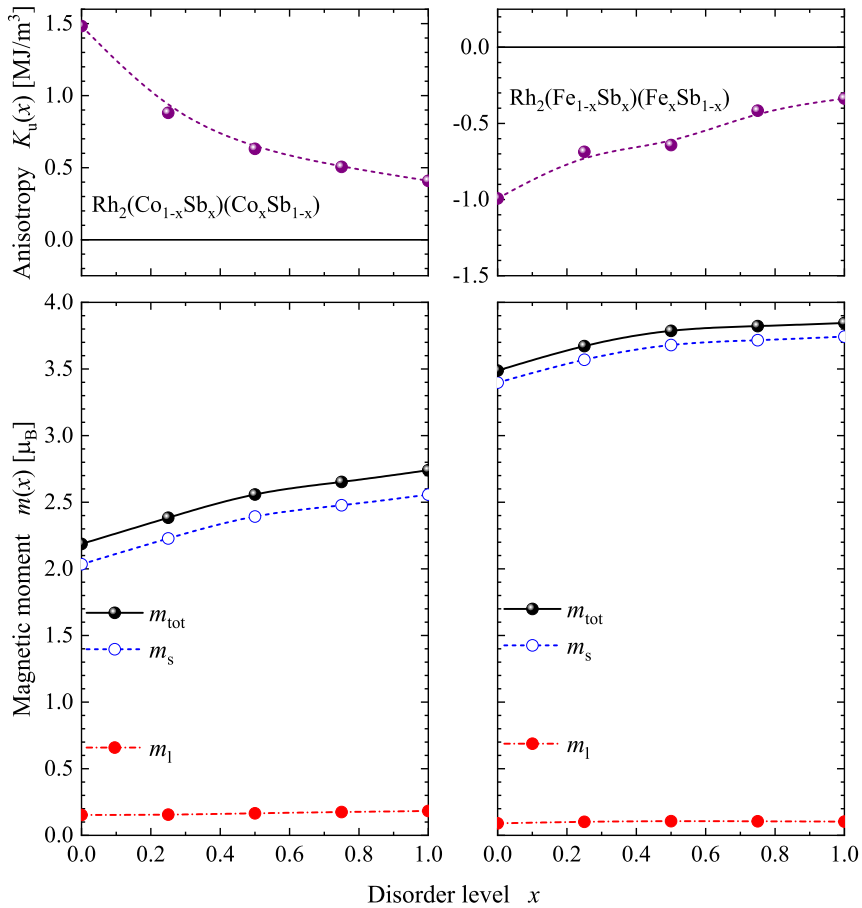

FIG. 16. Disorder-induced changes in magnetic moments and anisotropy of $\mathrm{Rh}_{2}\left(T_{1-x / 2} \mathrm{Sb}_{x / 2}\right)\left(T_{x / 2} \mathrm{Sb}_{1-x / 2}\right) T=\mathrm{Co}, \mathrm{Fe}$. Total, spin, and orbital magnetic moments are shown, along with the uniaxial anisotropy constant $K_{u}$ as a function of disorder level $x$.

\section{Off-stoichiometric alloys}

In many experiments, 2:1:1 stoichiometry was not fully reached, but an excess of $\mathrm{Fe}$ or $\mathrm{Co}$ and a deficiency of $\mathrm{Sb}$ was obtained. In particular, the magnetic properties of $\mathrm{Rh}_{2} T_{1+x} \mathrm{Sb}_{1-x}$, with $T=\mathrm{Fe}$, Co, were calculated for $x=$ 0.12 . As in the study of disorder, the calculations were performed using the CPA. The $2 a$ site is assumed to be occupied by $12 \%$ with $\mathrm{Fe}$ (or $\mathrm{Co}$ ) and by $88 \%$ with $\mathrm{Sb}$, whereas the occupations of the $4 d$ and $2 b$ sites are unchanged.

The calculated magnetic properties of the offstoichiometric alloys are listed in Table V. The magnetic moments and spin stiffness $D_{0}$ are enhanced in both alloys, and the values are higher than those of the stoichiometric compound. In particular, the excess $\mathrm{Co}$ and $\mathrm{Fe}$ atoms on the $2 a$ site contribute a large spin moment. The total magnetic moment, $m_{s}+m_{l}=2.627 \mu_{B}$, of $\mathrm{Rh}_{2} \mathrm{Co}_{1.12} \mathrm{Sb}_{0.88}$ is very similar to the experimentally observed value of $2.6 \mu_{B}$. The Curie temperature of the off-stoichiometric Co-containing compound is slightly lower, whereas that of the Fe compound is slightly higher. These findings, along with the spin stiffness results, suggest that the exchange coupling parameters of the stoichiometric compounds differ from those of the off-stoichiometric alloys.

The type of anisotropy (easy plane or easy axis) is the same in the off-stoichiometric alloys as in the stoichiometric compounds. The uniaxial anisotropy constants differ, however. They are enhanced in the Fe alloy and reduced in the Co alloy.
TABLE V. Calculated magnetic properties of off-stoichiometric $\mathrm{Rh}_{2} T_{1.12} \mathrm{Sb}_{0.88}$. Spin $m_{s}$ and orbital $m_{l}$ magnetic moments per atom $(\mathrm{Rh}, \mathrm{Co}, \mathrm{Fe})$ and those of the primitive cell (tot al) are listed, as well as the Curie temperature $T_{C}$ and spin stiffness $D_{0} . m_{s, l}^{2 \mathrm{~b}}$ represents the magnetic moments at the original position, and $m_{s, l}^{2 a}$ represents the moments of the excess $\mathrm{Fe}$ and $\mathrm{Co}$ atoms at the initial $\mathrm{Sb}$ position.

\begin{tabular}{lcc}
\hline \hline $\mathrm{Rh}_{2} T_{1.12} \mathrm{Sb}_{0.88}$ & $T=\mathrm{Fe}$ & $T=\mathrm{Co}$ \\
\hline$m_{s}^{\mathrm{Rh}}\left(\mu_{B}\right)$ & 0.308 & 0.244 \\
$m_{l}^{\mathrm{Rh}}\left(\mu_{B}\right)$ & 0.012 & 0.009 \\
$m_{s}^{2 \mathrm{~b}}\left(\mu_{B}\right)$ & 2.988 & 1.691 \\
$m_{l}^{2 \mathrm{~b}}\left(\mu_{B}\right)$ & 0.092 & 0.140 \\
$m_{s}^{2 \mathrm{a}}\left(\mu_{B}\right)$ & 3.564 & 2.521 \\
$m_{l}^{2 \mathrm{a}}\left(\mu_{B}\right)$ & 0.072 & 0.155 \\
$m_{s}^{\text {tot }}\left(\mu_{B}\right)$ & 4.010 & 2.453 \\
$m_{l}^{\text {tot }}\left(\mu_{B}\right)$ & 0.124 & 0.174 \\
$T_{C}(\mathrm{~K})$ & 490 & 480 \\
$D_{0}\left(\mathrm{meV} \AA^{2}\right)$ & 690 & 1100 \\
$K_{u}\left(\mathrm{MJ} / \mathrm{m}^{3}\right)$ & -1.667 & 0.826 \\
\hline \hline
\end{tabular}

\section{APPENDIX C: SEMIRELATIVISTIC BAND STRUCTURES}

The semirelativistic band structures of $\mathrm{Rh}_{2} \mathrm{FeSb}$ and $\mathrm{Rh}_{2} \mathrm{CoSb}$ are compared in Fig. 17 to illustrate the spin characteristics of the bands. The band structures are similar; the main differences result from the larger band filling in the Co-based compound, which has one more valence electron than the Fe compound. Further, the larger spin splitting in the $\mathrm{Fe}$ compound clearly results in a large spin magnetic moment.
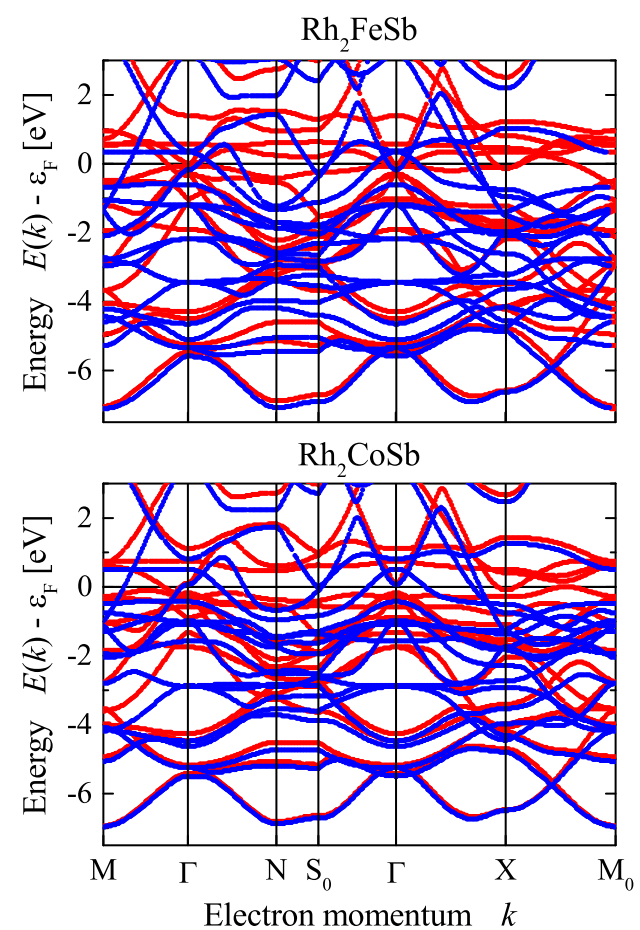

FIG. 17. Semirelativistic band structures of $\mathrm{Rh}_{2} \mathrm{FeSb}$ and $\mathrm{Rh}_{2} \mathrm{CoSb}$. Red and blue indicate majority and minority states, respectively. 


\section{APPENDIX D: MAGNETOCRYSTALLINE ANISOTROPY}

In this Appendix, the discussion of the magnetocrystalline anisotropy is extended beyond simple uniaxial approximations. The magnetocrystalline energy of uniaxial systems can be derived from the first-principles total energies for different magnetization directions (quantisation axes).

Textbooks give different descriptions of the magnetic anisotropy, in particular, different equations for the anisotropy constants [24-29]. Therefore, care must be taken when comparing the results of this paper with those of other studies or comparing other studies with each other.

\section{Uniaxial magnetic anisotropy}

It is often assumed that the magnetocrystalline anisotropy in tetragonal or hexagonal systems is simply described by a second-order dependence on the angle $\theta$ between the $c$ axis and the magnetization direction, that is,

$$
K_{u} \sin ^{2}(\theta)
$$

where $K_{u}$ is the uniaxial anisotropy constant. In that case,

$$
K_{u}=E^{100}-E^{001}
$$

is simply calculated from the difference between the energies for magnetization along the principal axes, $c \|[001]$ and $a \|[100]$. For $K_{u}>0$, the easy axis is along the $c$ axis, whereas $K_{u}<0$ describes an easy plane where $c$ is the hard axis. For a distinct magnetic anisotropy in the $a b$ plane, it would be more accurate to use the lowest energy of the two in-plane directions along the principal axis and the diagonal, which are the [100] and [110] directions, respectively:

$$
K_{u}=\min \left(E^{100}, E^{110}\right)-E^{001} .
$$

Equation (D1) has another serious drawback; namely, the anisotropy is completely independent of the crystal lattice, and the anisotropic energy distribution always has the same shape regardless of the $c / a$ parameter and whether the crystal has tetragonal, hexagonal, or some other structure. That is, Eq. (D1) is ultimately useful only for distinguishing between easy and hard $c$ axes.

Now we consider only tetragonal systems. By using the series expansion $\sum K_{2 v, 0} \sin ^{2 v}(\theta)$ up to the fourth order in $\sin (\theta)$, the uniaxial magnetocrystalline energy is expressed as

$$
E_{\text {crys }}^{\text {uniaxial }}=K_{0}+K_{2} \sin ^{2}(\theta)+K_{4} \sin ^{4}(\theta) .
$$

The equations for the sixth-order uniaxial anisotropy are discussed by Jensen and Bennemann [30], for example. In the following, the subscript crys is omitted, and the energies are indexed only by direction or by uni. For the high-symmetry directions $[h, k, l]$ and the lowest indices $(h, k, l=0,1)$, the energies depend on the anisotropy coefficients as follows:

$$
\begin{aligned}
& E^{001}=K_{0}, \\
& E^{100}=K_{0}+K_{2}+K_{4}, \text { or } \\
& E^{110}=K_{0}+K_{2}+K_{4}, \text { and } \\
& E^{101}=K_{0}+K_{2} \sin ^{2}\left(\theta^{101}\right)+K_{4} \sin ^{4}\left(\theta^{101}\right), \text { or } \\
& E^{111}=K_{0}+K_{2} \sin ^{2}\left(\theta^{111}\right)+K_{4} \sin ^{4}\left(\theta^{111}\right) .
\end{aligned}
$$

Note that the energies for the [100] and [110] directions are identical only when uniaxial anisotropy is assumed. The energies for the [101] and [111] directions, however, have different angles with respect to the $c$ axis. From Eqs. ((D4) and (D5)), $K_{2}$ and $K_{4}$ may be obtained, for example, from the differences:

$$
\begin{aligned}
& E^{100}-E^{001}=K_{2}+K_{4} \text { and } \\
& E^{101}-E^{001}=K_{2} \sin ^{2}(\theta)+K_{4} \sin ^{4}(\theta) .
\end{aligned}
$$

For $z=c / a$, the angle $\theta$ is found using $\theta^{101}=\theta^{011}=$ $\arctan (1 / z)$. From Eq. (D5) or (D6), the anisotropy constants $K_{i}$ are given by

$$
\begin{aligned}
& K_{0}=E^{001}, \\
& K_{2}=\left(E^{101}-E^{001}\right)\left(z^{2}+2\right)+\left(E^{101}-E^{100}\right) \frac{1}{z^{2}}, \\
& K_{4}=\left(E^{001}-E^{101}\right)\left(z^{2}+1\right)+\left(E^{100}-E^{101}\right) \frac{\left(z^{2}+1\right)}{z^{2}} .
\end{aligned}
$$

Alternatively, $E^{111}$ and $\theta^{111}=\arctan (\sqrt{2} / z)$ may be used, but the resulting equations will have a different dependence on $c / a$. The uniaxial magnetocrystalline anisotropy energy $\left(E_{u}\right)$ is the difference between the magnetocrystalline energy (here $E^{\text {uni }}$ ) and the isotropic contribution, which is the spherical part $K_{0}$ :

$$
E_{u}=E^{\mathrm{uni}}-K_{0} .
$$

According to this equation, the uniaxial magnetocrystalline anisotropy energy may be positive or negative, depending on the directions and values of $K$ (see also Appendix D 4).

Equation (D4) has four extremal values at

$$
\theta_{i}=0, \frac{\pi}{2}, \text { and } \pm \arcsin \left(\sqrt{\frac{-K_{2}}{2 K_{4}}}\right),
$$

where the first derivative of the fourth-order Equation (D4) vanishes, that is, for $d E^{\text {uniaxial }} / d \theta=0$. The solutions $\theta_{3,4}$ are real only if the anisotropy constants obey the relation $0 \leqslant$ $\frac{-K_{2}}{2 K_{4}} \leqslant 1$, that is, $K_{2} K_{4} \geqslant 0,\left|K_{2}\right| \leqslant 2\left|K_{4}\right|$. For $K_{2}=-2 K_{4}$, one has $\theta_{3,4}= \pm 90^{\circ}$. For a real $\theta_{3,4}$, one has an easy or a hard cone. The resulting extremal energies are

$$
\begin{aligned}
E(0) & =K_{0}, \\
E(\pi / 2) & =K_{0}+K_{2}+K_{4}, \\
E\left(\theta_{3,4}\right) & =K_{0}-\frac{K_{2}^{2}}{4 K_{4}} .
\end{aligned}
$$

The minima or maxima are obtained using the second derivatives of the energy at the extremal angles:

$$
\begin{aligned}
\left.\frac{d^{2} E}{d \theta^{2}}\right|_{0} & =2 K_{2}, \\
\left.\frac{d^{2} E}{d \theta^{2}}\right|_{\pi / 2} & =-2\left(K_{2}+2 K_{4}\right), \\
\left.\frac{d^{2} E}{d \theta^{2}}\right|_{\theta_{3,4}} & =-\frac{2 K_{2}\left(K_{2}+2 K_{4}\right)}{K_{4}} .
\end{aligned}
$$

The minima appear for positive second derivatives $\left(d^{2} E /\left.d \theta^{2}\right|_{\theta_{i}}>0\right)$ and define the easy direction(s) of magne- 
TABLE VI. Uniaxial anisotropy phase diagram. $a b$ stands for basal plane, $c$ stands for $c$ axis. Cones may have an opening angle $\theta$ or $\pi / 4$ with respect to the $c$ axis.

\begin{tabular}{cccc}
\hline \hline$K_{2}$ & $K_{4}$ & Easy & Hard \\
\hline$>0$ & $-\infty \cdots-K_{2}$ & $a b$ & cone $(\theta)$ \\
$>0$ & $-K_{2}$ & $a b, c$ & cone $\left(45^{\circ}\right)$ \\
$>0$ & $-K_{2} \cdots-K_{2} / 2$ & $c$ & cone $(\theta)$ \\
$>0$ & $-K_{2} / 2 \cdots \infty$ & $c$ & $a b$ \\
$=0$ & $=K_{2}=0$ & undefined, spherical \\
$<0$ & $-K_{2} \cdots \infty$ & cone $(\theta)$ & $a b$ \\
$<0$ & $-K_{2}$ & cone $\left(45^{\circ}\right)$ & $a b, c$ \\
$<0$ & $-K_{2} / 2 \cdots-K_{2}$ & cone $(\theta)$ & $c$ \\
$<0$ & $-\infty \cdots-K_{2} / 2$ & $a b$ & $c$ \\
\hline \hline
\end{tabular}

tization. Indeed, one has to search for the absolute minimum and maximum to find the correct easy and hard axes, planes, or cones. An easy cone appears for $K_{2}<0, K_{4}>-K_{2} / 2$, and the corresponding cone angle is given by $\theta_{3,4}$. A special hard cone exists for $K_{2}>0, K_{4}=-K_{2}$, where both the $c$ axis and the $a b$ plane have the same (lowest) energy. The energy barrier at the hard cone must be overcome, however, to change the magnetization direction from the easy axis to the easy plane and vice versa. In the range $-\infty<K_{4}<-K_{2} / 2$, the solutions are metastable when $K_{2}>0$. The complete fourth-order uniaxial anisotropy phase diagram is presented in Table VI.

The magnetic anisotropy phase diagram for fourth-order uniaxial anisotropy is displayed in Fig. 18. It is similar to the graphical representations reported in Refs. [30,31]. The different phases are distinguished. For $K_{4}=-K_{2}<0$, there is a distinct metastable case with equal energies for magnetization along the $c$ axis and in the $a b$ plane. At this line, a transition occurs from easy-axis to easy-plane

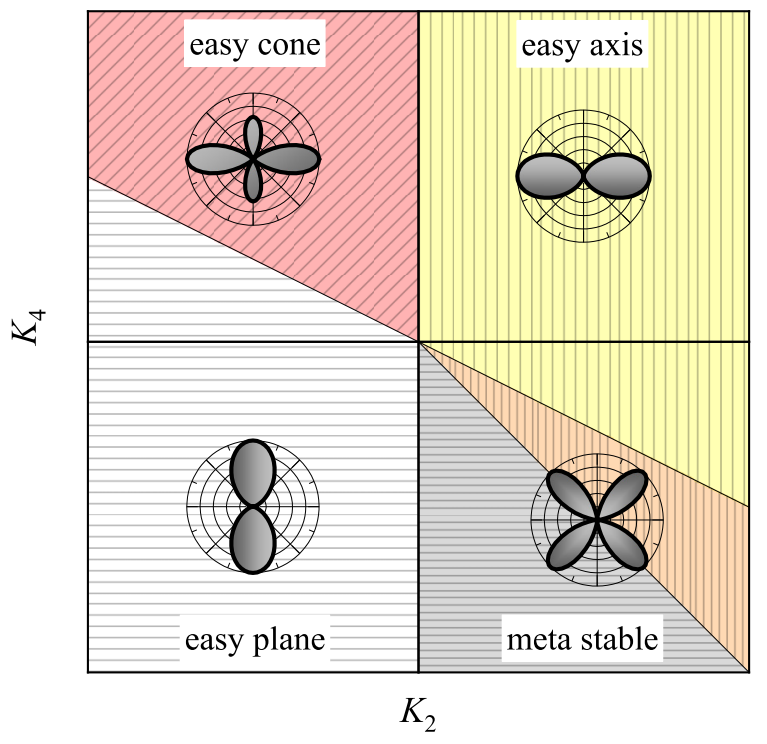

FIG. 18. Magnetic anisotropy phase diagram. In the metastability range, hard-cone-type anisotropy occurs. In the sketches of the $E(\theta)$ distributions, it is assumed that $K_{0}=0$. behavior. In the metastable region for $K_{4}<-K_{2} / 2<0$, easyaxis behavior appears, whereas easy-plane behavior appears for $K_{4}<-K_{2}<0$ (see Table VI). In both cases, the sizes of the anisotropy constants determine how easily one state can switch to the other and the stability of the state with lower energy. The energy barrier to cross the hard cone has a size of $-\frac{K_{2}^{2}}{4 K_{4}}$, as mentioned above.

\section{Tetragonal magnetic anisotropy}

The uniaxial magnetic anisotropy does not reflect the symmetry of the crystal structure. The symmetry of the anisotropy should generally be the same as the symmetry of the crystal potential; thus, it is given by the fully symmetric irreducible representation of the point group, that is, $a, a_{1}, a_{g}$, or similar. Again, by using a series expansion up to the fourth order in $\sin (\theta)$, the magnetocrystalline energy of a tetragonal system is expressed as

$$
\begin{aligned}
E_{\text {crys }}^{\text {tetragonal }} & =\sum_{\nu=0}^{2} K_{2 v, 0} \sin ^{2 v}(\theta)+K_{4,4} \sin ^{4}(\theta) f(\phi), \\
& =E_{\text {crys }}^{\text {uniaxial }}+K_{4,4} \sin ^{4}(\theta) f(\phi), \\
f(\phi) & =\cos (4 \phi) .
\end{aligned}
$$

$f(\phi)$ has an azimuthal dependence on $4 \phi$, which results in the expected fourfold symmetry. Some works used $f^{\prime}(\phi)=$ $\sin ^{4}(\phi)+\cos ^{4}(\phi)$, which results in different equations and $K$ values. Higher-order approximations will include terms with $K_{6,0}, K_{6,4}, K_{8,0}, K_{8,4}, K_{8,8}$, and so on. Subtracting $K_{0}$ from Eq. (D12) yields

$$
E_{a}(\vec{r})=K_{2,0} \sin ^{2}(\theta)+\left[K_{4,0}+K_{4,4} \cos (4 \phi)\right] \sin ^{4}(\theta) .
$$

In the following, the subscript crys is omitted, and the energies are indexed only by direction or by tet. For the highsymmetry directions $[h, k, l]$ and the lowest indices $(h, k, l=$ $0,1)$, Eq. (D12) gives

$$
\begin{aligned}
& E^{001}=K_{0,0}, \\
& E^{100}=K_{0,0}+K_{2,0}+K_{4,0}+K_{4,4}, \\
& E^{110}=K_{0,0}+K_{2,0}+K_{4,0}-K_{4,4}, \text { and } \\
& E^{101}=\sum_{\nu=0,2} K_{2 v, 0} \sin ^{2 v}\left(\theta^{101}\right)+K_{4,4} \sin ^{4}\left(\theta^{101}\right), \text { or } \\
& E^{111}=\sum_{\nu=0,2} K_{2 v, 0} \sin ^{2 v}\left(\theta^{111}\right)-K_{4,4} \sin ^{4}\left(\theta^{111}\right) .
\end{aligned}
$$

For $z=c / a$, the angle $\theta^{101}$ is found using $\theta^{101}=\theta^{011}=$ $\arctan (1 / z)$. Alternatively, $E^{111}$ with $\theta^{111}=\arctan (\sqrt{2} / z)$ may be used. From the first four energies of Eq. (D13), the anisotropy constants $K_{l, m}$ are found to be

$$
\begin{aligned}
K_{0,0}= & E^{001}, \\
K_{2,0}= & \left(E^{101}-E^{001}\right)\left(z^{2}+2\right) \\
& +\left(E^{101}-E^{100}\right) \frac{1}{z^{2}}, \\
K_{4,0}= & \left(E^{001}-E^{101}\right)\left(z^{2}+1\right)
\end{aligned}
$$




$$
\begin{aligned}
& +\left(E^{100}-E^{101}\right) \frac{1}{z^{2}} \\
& +\frac{1}{2}\left(E^{100}+E^{110}-2 E^{101}\right), \\
K_{4,4}= & \frac{1}{2}\left(E^{100}-E^{110}\right) .
\end{aligned}
$$

The magnetocrystalline anisotropy energy $\left(E_{a}\right)$ is the difference between the magnetocrystalline energy (here $E^{\text {tet }}$ ) and the isotropic contribution, which is the spherical part $K_{0}$ :

$$
E_{a}=E^{\text {tet }}-K_{0}
$$

\section{Dipolar magnetic anisotropy}

In noncubic systems, the dipolar anisotropy does not vanish and also contributes to the magnetocrystalline anisotropy. It is calculated from a direct lattice sum yielding the dipolar energy,

$$
E_{\mathrm{dip}}(\vec{n})=\frac{\mu_{0}}{8 \pi} \sum_{i \neq j}\left[\frac{\vec{m}_{i} \cdot \vec{m}_{j}}{r_{i j}^{3}}-3 \frac{\left(\vec{r}_{i j} \cdot \vec{m}_{i}\right)\left(\vec{r}_{i j} \cdot \vec{m}_{j}\right)}{r_{i j}^{5}}\right],
$$

where $\vec{n}=\vec{M} / M$ is the magnetization direction, and $r_{i j}$ represents the distance vectors between the magnetic moments $m_{i}$ and $m_{j}$. The individual magnetic moments, $\vec{m}_{i}$ and $\vec{m}_{j}$, do not necessarily have to be collinear in general.

In a simplified picture, only the $3 d$ transition elements $T$ carry a significant magnetic moment in the $\mathrm{Rh}_{2} T \mathrm{Sb}$ compounds investigated here. In all cases of a single magnetic ion where all the magnetic moments in the structure are collinear along $\vec{n}$, the equation simplifies to

$$
\begin{aligned}
E_{\mathrm{dip}}(\vec{n}) & =\frac{\mu_{0} m^{2}(\vec{n})}{8 \pi} \sum_{i \neq j} \frac{1}{r_{i j}^{3}}\left[1-3 \frac{r_{n, i j}^{2}}{r_{i j}^{2}}\right], \\
& =\frac{\mu_{0} m^{2}(\vec{n})}{8 \pi} \sum_{i \neq j} \frac{1-3 \cos ^{2}\left(\theta_{i j}\right)}{r_{i j}^{3}},
\end{aligned}
$$

where $r_{n, i j}=r_{n, i j}(\vec{n})$ is a projection of the position vector onto the direction of the magnetic moment, and $\theta_{i j}$ is the angle between them. In Eq. (D17), the sign of the energy is completely defined by the crystal structure when the summation is over a spherical particle. Note that the size of the magnetic moment, $m(\vec{n})$, depends on the magnetization direction when the spin-orbit interaction is taken into account.

Finally, the dipolar anisotropy is given by the difference between the energies for two different directions, $\Delta E_{\text {dipaniso }}=$ $E\left(\vec{n}_{2}\right)-E\left(\vec{n}_{1}\right)$. Again, the two well-distinguished directions are the $\vec{n}_{1}=$ [001] and $\vec{n}_{2}=$ [100] directions, which are along the $c$ axis and in the basal plane along $a$, respectively. Positive values indicate an easy dipolar direction that is along the [001] axis. It has a second-order angular dependence.

\section{Plotting the magnetic anisotropy}

According to Eqs. (D8) and (D15), the magnetocrystalline anisotropy energy may be positive or negative, depending on the direction of $(\theta, \phi)$ and the $K$ values. Consequently, it is difficult to visualise the anisotropy energy by plotting the three-dimensional distribution of $E_{a}(\vec{r})=E_{a}(\theta, \phi)$. Therefore, the alternative anisotropy energy $E_{a^{\prime}}$ with respect to the lowest energy is generally plotted, where

$$
E_{a^{\prime}}=E_{a}-\min \left(E_{a}\right),
$$

which is still positive even when $E_{a}<0$. The easy directions or planes are identified as those for which $E_{a^{\prime}}=0 . E_{a^{\prime}}$ is used to plot the magnetocrystalline anisotropy in the main text.
[1] S. K. Dhar, A. K. Grover, S. K. Malik, and R. Vijayaraghavan, Peaks in low field a.c. susceptibility of ferromagnetic Heusler alloys, Solid State Commun. 33, 545 (1980).

[2] S. V. Faleev, Y. Ferrante, J. Jeong, M. G. Samant, B. Jones, and S. S. P. Parkin, Heusler compounds with perpendicular magnetic anisotropy and large tunneling magnetoresistance, Phys. Rev. Mater. 1, 024402 (2017).

[3] Y. He, G. H. Fecher, C. Fu, Y. Pan, K. Manna, J. Kroder, A. Jha, X. Wang, Z. Hu, S. Agrestini, J. Herrero, M. Valvidares, Y. Skourski, W. Schnelle, P. Stamenov, H. Borrmann, L.-H. Tjeng, R. Schaefer, S. S. P. Parkin, J. M. D. Coey et al., New highly-anisotropic Rh-based Heusler compound for magnetic recording, Adv. Mater. 32, 2004331 (2020).

[4] See Supplemental Material at https://link.aps.org/supplemental/ 10.1103/PhysRevMaterials.5.054404 for results from experiments.

[5] P. Blaha, K. Schwarz, P. Sorantin, and S. B. Trickey, Fullpotential, linearized augmented plane wave programs for crystalline systems, Comput. Phys. Commun. 59, 399 (1990).
[6] K. Schwarz and P. Blaha, Solid state calculations using WIEN2k, Comput. Mater. Sci. 28, 259 (2003).

[7] P. Blaha, K. Schwarz, G. K. H. Madsen, D. Kvasnicka, and J. Luitz, WIEN2k: An Augmented PlaneWave + Local Orbitals Program for Calculating Crystal Properties (Techn. Universitat, Vienna, Austria, 2013).

[8] H. Ebert, Fully relativistic band structure calculations for magnetic solids-formalism and application, in Electronic Structure and Physical Properties of Solids. The Use of the LMTO Method, Lecture Notes in Physics, Vol. 535, edited by H. Dreysse (Springer-Verlag, Berlin, Heidelberg, 1999), pp. 191-246.

[9] H. Ebert, D. Ködderitzsch, and J. Minar, Calculating condensed matter properties using the KKR-Green's function methodrecent developments and applications, Rep. Prog. Phys. 74, 096501 (2011).

[10] J. P. Perdew, K. Burke, and M. Ernzerhof, Generalized Gradient Approximation Made Simple, Phys. Rev. Lett. 77, 3865 (1996).

[11] H. C. Kandpal, G. H. Fecher, and C. Felser, Calculated electronic and magnetic properties of the half-metallic, transition 
metal based Heusler compounds, J. Phys. D: Appl. Phys. 40, 1507 (2007).

[12] G. H. Fecher, S. Chadov, and C. Felser, Theory of the halfmetallic Heusler compounds, in Spintronics, edited by C. Felser and G. H. Fecher (Springer Verlag, Dordrecht, 2013), Chap. 7, p. 115.

[13] S. Mankovsky, G. H. Fecher, and H. Ebert, Electronic structure calculations in ordered and disordered solids with spiral magnetic order, Phys. Rev. B 83, 144401 (2011).

[14] J. Thoene, S. Chadov, G. Fecher, C. Felser, and J. Kübler, Exchange energies, Curie temperatures and magnons in Heusler compounds, J. Phys. D: Appl. Phys. 42, 084013 (2009).

[15] P. Soven, Coherent-potential model of substitutional disordered alloys, Phys. Rev. 156, 809 (1967).

[16] S. Ayaz Khan, P. Blaha, H. Ebert, J. Minar, and O. Sipr, Magnetocrystalline anisotropy of FePt: A detailed view, Phys. Rev. B 94, 144436 (2016).

[17] P. Villars and K. Cenzual, Pearson's Crystal DataCrystal Structure Database for Inorganic Compounds Release 2019/2020 (ASM International, The Materials Information Company, Materials Park, Ohio, 2020).

[18] S. J. Joshua, Symmetry Principles and Magnetic Symmetry in Solid State Physics (Adam Hilger, IOP Publishing Ltd., Bistol, Philadelphia, 1991).

[19] R. Lizarraga, L. Nordström, L. Bergqvist, A. Bergman, E. Sjöstedt, P. Mohn, and O. Eriksson, Conditions for Noncollinear Instabilities of Ferromagnetic Materials, Phys. Rev. Lett. 93, 107205 (2004).

[20] A. I. Liechtenstein, M. I. Katsnelson, and V. A. Gubanov, Exchange interactions and spin-wave stiffness in ferromagnetic metals, J. Phys. F: Met. Phys. 14, L125 (1984).
[21] A. I. Liechtenstein, M. I. Katsnelson, V. P. Antropov, and V. A. Gubanov, Local spin density functional approach to the theory of exchange interactions in ferromagnetic metals and alloys, $\mathrm{J}$. Magn. Magn. Mater. 67, 65 (1987).

[22] M. Pajda, J. Kudrnovsky, I. Turek, V. Drchal, and P. Bruno, Ab initio calculations of exchange interactions, spin-wave stiffness constants, and Curie temperatures of $\mathrm{Fe}, \mathrm{Co}$, and Ni, Phys. Rev. B 64, 174402 (2001).

[23] I. Turek, J. Kudrnovsky, and K. Carva, Magnetic anisotropy energy of disordered tetragonal Fe-Co systems from ab initio alloy theory, Phys. Rev. B 86, 174430 (2012).

[24] R. Skomsky and J. M. D. Coey, Permanent Magnetism, Studies in Condensed Matter Physics (Taylor and Francis Group, New York, 1999).

[25] J. Kübler, Theory of Itinerant Electron Magnetism (Clarendon Press, Oxford, 2000).

[26] B. D. Cullity and C. D. Graham, Introduction to Magnetic Materials, 2nd ed. (John Wiley and Sons, Hoboken, 2009).

[27] J. M. D. Coey, Magnetism and Magnetic Materials (Cambridge University Press, Cambridge, 2010).

[28] N. A. Spaldin, Magnetic Materials, 2nd ed., Fundamentals and Applications (Cambridge University Press, Cambridge, 2011).

[29] K. M. Krishnan, Fundamentals and Applications of Magnetic Materials (Oxford University Press, Oxford, 2016).

[30] P. J. Jensen and K. H. Bennemann, Magnetic structure of films: Dependence on anisotropy and atomic morphology, Surf. Sci. Rep. 61, 129 (2006).

[31] R. Skomski, H.-P. Oepen, and J. Kirschner, Unidirectional anisotropy in ultrathin transition-metal films, Phys. Rev. B 58, 11138 (1998). 